Pacific Journal of Mathematic 


\title{
ON A CLASS OF UNBOUNDED OPERATOR ALGEBRAS
}

\author{
ATSUShI INOUE
}

The primary purpose of this paper is to investigate the structures of functionals and homomorphisms of unbounded operator algebras called symmetric \#-algebras, $E C^{\#}$-algebras and $E W^{\sharp}$-algebras. First, we give the definitions and the fundamental properties of such algebras. In particular, we define several locally convex topologies on such algebras; a weak topology, a strong topology, a $\sigma$-weak topology and a $\sigma$-strong topology. In the next section, we study the elementary operations on $E W^{*}$-algebras. We can define induced and reduced $E W^{*}$-algebras, the product of $E W^{*}$-algebras and homomorphisms called an induction and an amplification. In the final two sections, we obtain the main results (Theorem 4.8 and 5.5) which are described here. It is shown that a linear functional $f$ on a closed $E W^{*}$-algebra $\mathfrak{A}$ on $\mathscr{D}$ is weakly continuous (resp. $\sigma$-weakly continuous) if and only if $f(A)=$ $\sum_{i=1}^{n}\left(A \xi_{i} \mid \eta_{i}\right), A \in \mathfrak{A} ; \xi_{i}, \eta_{i} \in \mathfrak{D}(i=1,2, \cdots, n)$ (resp. $f(A)=$ $\sum_{n=1}^{\infty}\left(A \xi_{n} \mid \eta_{n}\right) ; \xi_{n}, \quad \eta_{n} \in \mathcal{D}(n=1,2, \cdots)$ and $\sum_{n=1}^{\infty}\left\|T \xi_{n}\right\|^{2}<\infty$, $\sum_{n=1}^{\infty}\left\|T \eta_{n}\right\|^{2}<\infty$ for all $\left.T \in \mathfrak{U}\right)$. Also, it is shown that a $\sigma$-weakly continuous homomorphism of a closed $E W^{\sharp}$-algebra $\mathfrak{X}$ onto a closed $E W^{\sharp}$-algebra $\mathfrak{B}$ is decomposed in the following three types; a spatial isomorphism, an induction and an amplification.

1. Introduction. In [2], G. R. Allan defined a class of locally convex involution algebras called $G B^{*}$-algebras, and proved that, in the commutative case, a $G B^{*}$-algebra is algebraically isomorphic to an algebra of extended-complex-valued continuous functions on a compact Hausdorff space. After that, in [4], P. G. Dixon considered the noncommutative case and characterized $G B^{*}$-algebras as a certain class of algebras of closed operators on a Hilbert space. And so, it seems that we should study representations onto algebras of closed operators on Hilbert spaces as those of locally convex *-algebras. Hence, in the previous paper [9] the author studied representations of locally convex *-algebras onto algebras of closed operators on Hilbert spaces. In order to investigate such representations in detail, it seems that we should begin by studying a class of algebras of closed operators on Hilbert spaces. In this paper we study unbounded operator algebras called symmetric \#-algebras, $E C^{\sharp}$-algebras and $E W^{*}$-algebras. The author would like to thank Professors R. T. Powers and P. G. Dixon for giving him the basic ideas in $[4,5,9]$. 
2. Definitions and fundamental properties. For the definitions and the basic properties concerning unbounded representations (resp. locally convex $*$-algebras) the reader is referred to $[9,11]$ (resp. $[2,4])$.

Let $\pi$ be a closed *-representation on a Hilbert space $\mathfrak{H}_{\mathfrak{c}}$ of a pseudo-complete symmetric locally convex *-algebra $A$. Then $\pi(A)$ is an algebra of linear operators all defined on a common dense domain $\mathfrak{D}(\pi)$ in $\mathfrak{S}$ and we have

$$
(\pi(x) \xi \mid \eta)=\left(\xi \mid \pi\left(x^{*}\right) \eta\right)
$$

for all $\xi, \eta \in \mathfrak{D}(\pi)$ and $x \in A$, and $\left(I+\pi\left(x^{*}\right) \pi(x)\right)^{-1}$ exists and lies in $\pi(A)$, where $I$ is an identity operator on $\mathfrak{D}(\pi)$. On the basis of $\pi(A)$ we define a certain unbounded operator algebra.

Let $\mathfrak{D}$ be a pre-Hilbert space with inner product $(\mid)$ and let $\mathfrak{S}$ be the completion of $\mathfrak{D}$. We denote the set of all linear operators on $\mathfrak{D}$ by $\mathscr{L}(\mathfrak{D})$.

DEFINITION 2.1. Let $\mathfrak{A}$ be a subalgebra of $\mathscr{L}(\mathfrak{D})$ with an identity operator $I . \quad \mathscr{U}$ is called a symmetric \#-algebra on $\mathfrak{D}$ if the following conditions (1) and (2) are satisfied;

(1) There exists an involution on $\mathfrak{X} ; A \rightarrow A^{\sharp}$ such that

$$
(A \xi \mid \eta)=\left(\xi \mid A^{\sharp} \eta\right)
$$

for all $A \in \mathfrak{X}$ and $\xi, \eta \in \mathfrak{D}$,

(2) $\left(I+A^{\sharp} A\right)^{-1}$ exists and lies in $\mathfrak{A}_{b}$ for all $A \in \mathfrak{A}$, where let $\mathfrak{A}_{b}$ be the set of all bounded operators in $\mathfrak{A}$.

Let $\mathfrak{U}$ be a symmetric \#-algebra on $\mathfrak{D}$. Each $A$ in $\mathfrak{U}$ is a closable operator on $\mathfrak{S}$ and hence we denote the closure of $A$ by $\bar{A}$ and put $\overline{\mathfrak{A}}=\{\bar{A} ; A \in \mathfrak{Q}\}$.

Definition 2.2. Let $\mathfrak{U}$ be a symmetric $\#$-algebra on $\mathfrak{D}$. If $\overline{\mathfrak{A}}_{b}$ is a $C^{*}$-algebra (resp. $W^{*}$-algebra), then $\mathfrak{U}$ is said to be an $E C^{*}$-algebra (resp. $E W^{*}$-algebra).

REMARK. If $\mathfrak{A}$ is an $E C^{\sharp}$-algebra (resp. $E W^{\sharp}$-algebra) on $\mathfrak{D}$, then $\overline{\mathfrak{A}}$ becomes an $E C^{*}$-algebra (resp. $E W^{*}$-algebra) defined by P. G. Dixon [5].

Let $S, T$ be closed operators on a Hilbert space $\mathfrak{S}$. If $S+T$ is closable, then $\overline{S+T}$ is called the strong sum of $S$ and $T$, and is denoted $S+T$. The strong product is likewise defined to be $\overline{S T}$, if it exists, and is denoted $S \cdot T$. The strong scalar multiplication of $\lambda \in \mathbb{C}$ (당 the field of complex numbers) and $T$ is defined by $\lambda \cdot T=$ $\lambda T$ if $\lambda \neq 0$ and $\lambda \cdot T=0$, if $\lambda=0$. 
THEOREM 2.3. Let $\mathfrak{A}$ be a symmetric \#-algebra on $\mathfrak{D}$. Then we have

$$
\bar{A}+\bar{B}=\overline{A+B}, \bar{A} \cdot \bar{B}=\overline{A B}, \lambda \cdot \bar{A}=\overline{\lambda A}, \bar{A}^{*}=\bar{A}^{\sharp},
$$

for all $A, B \in \mathfrak{Y}$ and $\lambda \in \mathfrak{E}$. Therefore $\overline{\mathfrak{A}}$ is a *-algebra of closed operators under the operations of strong sum, strong product, adjoint and strong scalar multiplication and furthermore $\left(\bar{I}+\bar{A}^{*} \bar{A}\right)^{-1}$ exists and lies in $\overline{\mathfrak{A}}_{b}$ for all $A \in \mathfrak{N}$.

Proof. We shall show that $\bar{A}^{*}=\bar{A}^{\sharp}$ for every $A \in \mathfrak{2}$. Suppose $A^{\sharp}=A$. Then $\left(I+A^{2}\right)^{-1} \in \mathfrak{U}_{b}$ and we have

$$
A^{2}\left(I+A^{2}\right)^{-2}=\left(\left(I+A^{2}\right)-I\right)\left(I+A^{2}\right)^{-2}=\left(I+A^{2}\right)^{-1}-\left(I+A^{2}\right)^{-2}
$$

and hence $A^{2}\left(I+A^{2}\right)^{-2} \in \mathfrak{A}_{b}$. For each $\xi \in \mathfrak{D}$ we get $\left\|A\left(I+A^{2}\right)^{-1} \xi\right\|^{2} \leqq$ $\left\|\overline{A^{2}\left(I+A^{2}\right)^{-2}}\right\|\|\xi\|^{2}$, and so $A\left(I+A^{2}\right)^{-1} \in \mathfrak{A}_{b}$. Furthermore we have

$$
\begin{aligned}
& (i I-A)(-i I-A)\left(I+A^{2}\right)^{-1} \\
& \quad=(i I-A)\left\{-i\left(I+A^{2}\right)^{-1}-A\left(I+A^{2}\right)^{-1}\right\}=I
\end{aligned}
$$

and

$$
\left\{-i\left(I+A^{2}\right)^{-1}-A\left(I+A^{2}\right)^{-1}\right\}(i I-A)=I .
$$

Therefore $(i I-A)^{-1}$ exists and lies in $\mathfrak{A}_{b}$. For each $\gamma=\alpha+\beta i \epsilon$ $\mathfrak{C}-\Re(\Re$; the field of real numbers) we have

$$
(\lambda I-A)=\beta\left\{i I-\frac{1}{\beta}(A-\alpha I)\right\}
$$

and therefore $(\lambda I-A)^{-1}$ exists and lies in $\mathfrak{N}_{b}$. Therefore $\overline{(\lambda I-A)^{-1}}=$ $(\lambda \bar{I}-\bar{A})^{-1}$ is bounded for all $\lambda \in \mathbb{C}-\Re$, i.e., $\bar{A}$ has a real spectrum. Furthermore, since $A^{*} \supset A^{*}=A, \bar{A}$ is hermitian. Therefore $\bar{A}$ is selfadjoint, i.e., we have $\bar{A}^{*}=\bar{A}=\bar{A}^{\sharp}$.

For each $A \in \mathfrak{X}$ we show that $\bar{A}^{*}=\bar{A}^{\sharp}$. Let $H_{1}=\bar{A}^{*} \bar{A}$ and $H_{2}=$ $\left(\left(A^{\sharp}\right)^{*}\right)^{*}\left(A^{\sharp}\right)^{*}$. Clearly we have $H_{1} \supset \overline{A^{\sharp} A}$ and $H_{2} \supset \overline{A^{\sharp} A}$. Since $\left(A^{\sharp} A\right)^{\sharp}=$ $A^{\sharp} A, \overline{A^{\sharp} A}$ is self-adjoint. Since self-adjoint operators are maximal, it follows that $H_{1}=H_{2}=\overline{A^{\sharp} A}$. Hence we have $\mathfrak{D}(\bar{A})=\mathfrak{D}\left(H_{1}^{1 / 2}\right)=$ $\mathfrak{D}\left(H_{2}^{1 / 2}\right)=\mathfrak{D}\left(\left(A^{\sharp}\right)^{*}\right)$. Therefore we get $\bar{A}=\left(A^{\sharp}\right)^{*}$, and so $\bar{A}^{*}=\bar{A}^{\sharp}$.

We shall that $\bar{A}+\bar{B}=\overline{A+B}$ for all $A$ and $B$ in $\mathfrak{N}$. Since $\bar{A}+\bar{B}$ $\overline{\bar{A}+\bar{B}}$, clearly $\overline{A+\bar{B}} \subset \bar{A}+\bar{B}$. Since $\bar{A}=\left(A^{\sharp}\right)^{*}$, we have

$$
\overline{\bar{A}+\bar{B}}=\left(\overline{\left.A^{\sharp}\right)^{*}+\left(B^{\sharp}\right)^{*}} \subset\left(A^{\sharp}+B^{\sharp}\right)^{*}=\left((A+B)^{\sharp}\right)^{*}=\overline{A+B} .\right.
$$

Similarly we can show that $\bar{A} \cdot \bar{B}=\overline{A B}$ and $\lambda \cdot \bar{A}=\overline{\lambda A}$. For all $A \in \mathfrak{A}$, since $\left(\bar{I}+\bar{A}^{*} \bar{A}\right)^{-1}=\overline{\left(I+A^{\ddagger} A\right)^{-1}}$ and $\left(I+A^{\sharp} A\right)^{-1} \in \mathfrak{N}_{b},\left(\bar{I}+\bar{A}^{*} \bar{A}\right)^{-1}$ lies in $\overline{\mathfrak{A}}_{b}$. 
Let $\mathfrak{A}$ be a symmetric \#-algebra on $\mathfrak{D}$. Then there is a natural induced topology $\tau_{0}$ on $\mathfrak{D}$. This topology is defined as follows. Suppose that $\mathfrak{S}$ is a finite subset of elements of $\mathfrak{A}$. We define the seminorm \|\|$_{\widetilde{s}}$ on $\mathfrak{D}$ as

$$
\|\xi\|_{\mathcal{S}}=\sum_{A \in \mathcal{S}}\|A \xi\|
$$

where $\|\xi\|$ is the Hilbert space norm of $\xi$. We define the induced topology $\tau_{0}$ on $\mathfrak{D}$ as the topology generated by the family $\left\{\|\|_{\mathfrak{S}}\right.$; $S$ \} of the seminorms.

DEFINITION 2.4. Let $\mathfrak{A}$ be a symmetric \#-algebra on $\mathfrak{D}$. If $\mathfrak{D}$ is complete under the topology $\tau_{0}$, then $\mathfrak{A}$ is said to be closed.

Proposition 2.5. Let $\mathfrak{A}$ be a symmetric \#-algebra on $\mathfrak{D}$. Let

$$
\widetilde{D}=\bigcap_{A \in \mathfrak{r}} \mathfrak{D}(\bar{A}), \widetilde{A} x=\bar{A} x,(x \in \widetilde{\mathfrak{D}}) .
$$

Then $\tilde{\mathfrak{A}}=\{\tilde{A} ; A \in \mathfrak{N}\}$ is a closed symmetric \#-algebra on $\widetilde{\mathfrak{D}}$ and a minimal closed extension of $\mathfrak{A}$. Hereafter we call $\tilde{\mathfrak{U}}$ the closure of $\mathfrak{A}$.

Proof. By a slight modification of ([11] Lemma 2.6). Proposition 2.5 is easily shown.

Proposition 2.6. If $\mathfrak{U}$ is a closed symmtric \#-algebra on $\mathfrak{D}$, then we have

$$
\mathfrak{D}=\bigcap_{A \in \mathfrak{I}} \mathfrak{D}(\bar{A})=\bigcap_{A \in \mathfrak{I}} \mathfrak{D}\left(A^{*}\right) \text {. }
$$

Proof. By Proposition 2.5 we get $\mathfrak{D}=\bigcap_{A \in \mathfrak{x}} \mathfrak{D}(\bar{A})$. Since $A^{*}=$ $\bar{A}^{\sharp}$ for all $A \in \mathfrak{N}$, we have

$$
\bigcap_{A \in \mathfrak{I}} \mathfrak{D}\left(A^{*}\right)=\bigcap_{A \in \mathscr{T}} \mathfrak{D}\left(\bar{A}^{\sharp}\right)=\bigcap_{A \in \mathscr{I}} \mathfrak{D}(\bar{A})=\mathfrak{D} .
$$

We define several locally convex topologies in a symmetric \#algebra $\mathfrak{A}$ on $\mathfrak{D}$.

(1) The weak topology. The locally convex topology, induced by the seminorms;

$$
T \in \mathfrak{X} \longrightarrow P_{\xi, \eta}(T)=|(T \xi \mid \eta)|
$$

for each $\xi, \eta \in \mathfrak{D}$, is called the weak topology. Under the weak topology $\mathfrak{A}$ is a locally convex $\#$-algebra. Since

$$
\begin{aligned}
4(T \xi \mid \eta)= & (T(\xi+\eta) \mid \xi+\eta)-(T(\xi-\eta) \mid \xi-\eta) \\
& +i(T(\xi+i \eta) \mid \xi+i \eta)-i(T(\xi-i \eta) \mid \xi-i \eta),
\end{aligned}
$$


the weak topology is in accord with the topology induced by the seminorms $\left\{P_{\xi, \xi}() ; \xi \in \mathfrak{D}\right\}$.

If $\mathfrak{A}$ is an $E C^{\sharp}$-algebra on $\mathfrak{D}$, then $\overline{\mathfrak{A}}$ is a $G B^{*}$-algebra defined by P. G. Dixon [4] under the weak topology.

(2) The strong topology. The strong topology is the locally convex topology induced by the seminorms;

$$
T \in \mathfrak{A} \longrightarrow P_{\xi}(T)=\|T \xi\|, \xi \in \mathfrak{D} .
$$

(3) The $\sigma$-weak topology. Let

$$
\begin{gathered}
\mathfrak{D}_{\infty}(\mathfrak{U})=\left\{\xi_{\infty}=\left(\xi_{1}, \xi_{2}, \cdots, \xi_{n}, \cdots\right) ; \xi_{n} \in \mathfrak{D}, n=1,2, \cdots\right. \text { and } \\
\left.\sum_{n=1}^{\infty}\left\|T \xi_{n}\right\|^{2}<\infty \text { for all } T \in \mathfrak{U}\right\} .
\end{gathered}
$$

For each $\xi_{\infty}=\left(\xi_{1}, \xi_{2}, \cdots, \xi_{n}, \cdots\right)$ and $\eta_{\infty}=\left(\eta_{1}, \eta_{2}, \cdots, \eta_{n}, \cdots\right)$ in $\mathfrak{D}_{\infty}(\mathfrak{U})$, putting

$$
P_{\xi \infty, \eta_{\infty}}(T)=\left|\sum_{n=1}^{\infty}\left(T \xi_{n} \mid \eta_{n}\right)\right|, T \in \mathfrak{A},
$$

$P_{\xi \infty, \eta_{\infty}(}$ ) is a seminorm on $\mathfrak{A}$. We call the $\sigma$-weak topology in $\mathfrak{A}$ the locally convex topology in $\mathfrak{U}$ induced by the family $\left\{P_{\xi_{\infty}, \eta_{\infty}}(\quad) ; \xi_{\infty}\right.$, $\left.\eta_{\infty} \in \mathfrak{D}_{\infty}(\mathfrak{U})\right\}$ of seminorms. Under the $\sigma$-weak topology $\mathfrak{X}$ is a locally convex \#-algebra. The $\sigma$-weak topology is in accord with the topology induced by the seminorms $\left\{P_{\xi_{\infty}, \xi \infty}(\quad) ; \xi_{\infty} \in \mathfrak{D}_{\infty}(\mathfrak{I})\right\}$.

(4) The $\sigma$-strong topology. For each $\xi_{\infty}=\left(\xi_{1}, \xi_{2}, \cdots, \xi_{n}, \cdots\right) \epsilon$ $\mathfrak{D}_{\infty}(\mathfrak{R})$, putting

$$
P_{\xi \infty}(T)=\left(\sum_{n=1}^{\infty}\left\|T \xi_{n}\right\|^{2}\right)^{1 / 2}, \quad T \in \mathfrak{A},
$$

$P_{\xi \infty}()$ is a seminorm on $\mathfrak{2}$. The locally convex topology induced by the family $\left\{P_{\xi_{\infty}}(\quad) ; \xi_{\infty} \in \mathfrak{D}_{\infty}(\mathfrak{U})\right\}$ of seminorms is called the $\sigma$-strong topology in $\mathfrak{T}$.

DEFINITION 2.7. Let $\mathfrak{A}$ be a symmetric \#-algebra on $\mathfrak{D}$. We define the commutant $\mathfrak{X}^{\prime}$ of $\mathfrak{X}$ by

$$
\mathfrak{A}^{\prime}=\left\{C \in \mathscr{B}(\mathfrak{S}) ;(C A \xi \mid \eta)=\left(C \xi \mid A^{\sharp} \eta\right) \text { for all } A \in \mathfrak{N} \text { and } \xi, \eta \in \mathfrak{D}\right\} \text {, }
$$

where let $\mathscr{B}(\mathscr{S})$ be the set of all bounded linear operators on $\mathscr{S}$.

Proposition 2.8. Let $\mathfrak{A}$ be a (resp. closed) symmetric $\#$-algebra on $\mathfrak{D}$. Then $\mathfrak{X}^{\prime}$ is a von Neumann algebra and furthermore for each $C \in \mathfrak{X}^{\prime}$ we have $C \mathfrak{D} \subset \widetilde{D}$ (resp. $\left.C \mathfrak{D} \subset \mathfrak{D}\right)$ and $C A \xi=\widetilde{A} C \xi$ (resp. $C A \xi=$ $A C \xi)$ for all $A \in \mathfrak{A}$ and $\xi \in \mathfrak{D}$. 
Proof. This follows from ([11] Lemma 4.6).

Let $\mathfrak{A}$ be an $E W^{\sharp}$-algebra. Then we investigate the relations between the von Neumann algebra $\overline{\mathfrak{A}}_{b}$ and the von Neumann algebra $\mathfrak{U}^{\prime \prime}$.

Proposition 2.9. Let $\mathfrak{A}$ be an EC $E C^{\sharp}$-algebra on $\mathfrak{D}$. Then we have $\mathfrak{U}^{\prime}=\left(\overline{\mathfrak{A}}_{b}\right)^{\prime}$ and $\mathfrak{U}^{\prime \prime}=\left(\overline{\mathfrak{A}}_{b}\right)^{\prime \prime}$. In particular, if $\mathfrak{A}$ is an $E W^{*}$-algebra on $\mathfrak{D}$, then we have $\mathfrak{U}^{\prime \prime}=\overline{\mathfrak{A}}_{b}$.

Proof. Let $C \in \mathfrak{2}$. By Proposition 2.8 we have $C A \xi=\widetilde{A} C \xi$ for all $A \in \mathfrak{A}$ and $\xi \in \mathfrak{D}$. In particular, we have $C A \xi=\widetilde{A} C \xi$ for all $A \in \mathfrak{A}_{b}$ and hence $C \bar{A}=\bar{A} C$, i.e., $C \in\left(\overline{\mathfrak{A}}_{b}\right)^{\prime}$.

Conversely suppose that $C \in\left(\overline{\mathfrak{A}}_{b}\right)^{\prime}$. By ([5] Prop. 2.4) $\bar{A}$ is affiliated with $\left(\mathfrak{U}_{b}\right)^{\prime \prime}\left(\bar{A} \eta\left(\overline{\mathfrak{A}}_{b}\right)^{\prime \prime}\right)$ for every $A \in \mathfrak{N}$ and it follows that for each $\xi, \eta \in \mathfrak{D}$

$$
(C A \xi \mid \eta)=(\bar{A} C \xi \mid \eta)=\left(C \xi \mid A^{*} \eta\right)=\left(C \xi \mid A^{*} \eta\right) .
$$

Therefore we get $C \in \mathfrak{U}^{\prime}$.

DEFINITION 2.10. Let $\mathfrak{A}$ be a symmetric \#-algebra on $\mathfrak{D}$. An element $T$ of $\mathfrak{A}$ is called hermitian, if $T^{*}=T$ and we denote by $\mathfrak{A}_{h}$ the set of all hermitian elements of $\mathfrak{A}$. Let $T \in \mathfrak{N}_{h}$. If $(T \xi \mid \xi) \geqq 0$ for all $\xi \in \mathscr{D}$, then $T$ is called positive and write $T \geqq 0$. The set of all positive hermitian elements of $\mathfrak{U}$ is denoted $\mathfrak{U}_{h}^{+}$.

Proposition 2.11. Let $\mathfrak{i}$ be an EC $E C^{\sharp}$-algebra on $\mathfrak{D}$ and let $T \in \mathfrak{X}_{h}$. Then the following conditions are equivalent;

(1) $T \geqq 0$,

(2) $T=A^{2}$ for some $A \in \mathfrak{Q}_{h}^{+}$,

(3) $T=S^{\sharp} S$ for some $S \in \mathfrak{N}$,

(4) $\bar{T} \geqq 0$ (i.e., $(\bar{T} x \mid x) \geqq 0$ for every $x \in \mathfrak{D}(\bar{T})$ ).

Proof. If $\mathfrak{A}$ is an $E C^{\sharp}$-algebra, $\overline{\mathfrak{A}}$ is a $G B^{*}$-algebra under the weak topology. Therefore, by ([4] Prop. 5.1) and Theorem 2.3 we can easily prove the above proposition.

Proposition 2.12. Let $\mathfrak{X}$ be an EW $E W^{\sharp}$ algebra on $\mathfrak{D}$ and $T \in \mathfrak{X}$. Then there exist $U \in \mathfrak{Q}_{b}$ and $|T| \in \mathfrak{N}_{h}^{+}$such that $T=U|T|$, where $\bar{U}$ is a partial isometry whose initial domain is $\overline{\Re\left(T^{*}\right)}$ (we denote the range of $T$ by $\Re(T)$ ) and $\overline{|T|}$ is a positive self-adjoint operator such that $\mathfrak{R}(\overline{|T|})=\overline{\Re\left(T^{*}\right)}$. Furthermore such decomposition is unique.

Proof. By the polar decomposition of a closed operator $\bar{T}$, 
Theorem 2.3 and $\bar{T} \eta \overline{\mathfrak{A}}_{b}$ (Prop. 2.9) we can easily prove the above propositition.

Definition 2.13. The decomposition $T=U|T|$ of Proposition 2.12 is called the polar decomposition of $T$.

3. Elementary operations on $E W^{\sharp}$-algebras. We define reduced and induced $E W^{\sharp}$-algebras. Let $\mathfrak{U}$ be a symmetric $\#$-algebra on $\mathfrak{D}$. Define $\mathfrak{N}_{p}=\left\{E \in \mathfrak{N} ; E^{2}=E^{\ddagger}=E\right\}$ and let $E \in \mathfrak{A}_{p}$. For each $T \in \mathfrak{N}$. we define $T_{E}=E T / E D$ (the restriction of $E T$ onto $E(D)$ and $\mathfrak{V}_{L}=$ $\left\{T_{E} ; T \in \mathfrak{A}\right\}$. Then $T_{E}$ is a linear operator on $E \mathfrak{D}$. We put $\mathfrak{B}=$ $\{T \in \mathfrak{N} ; T E=E T=T\}$. Then $\mathfrak{B}$ is a $\#$-subalgebra of $\mathfrak{X}$ and we have $\mathfrak{B}=E \mathfrak{U} E$. The mapping $T \rightarrow T_{E}$ is an isomorphism of $\mathfrak{B}$ onto $\mathfrak{N}_{E}$.

THEOREM 3.1. Let $\mathfrak{A}$ be a symmetric \#-algebra on D. Suppose $E \in \mathfrak{X}_{p}$. Then $\mathfrak{R}_{E}$ is a smmetric \#-algebra on $E \mathfrak{D}$. In particular, if $\mathfrak{X}$ is an EW*-algebra on $\mathfrak{D}$, then $\mathfrak{N}_{E}$ is an $E W^{*}$-algebra on ED and we have

$$
\left(\mathfrak{U}_{E}\right)^{\prime}=\left(\mathfrak{Y}^{\prime}\right)_{\bar{E}}=\left(\left(\overline{\mathfrak{A}}_{b}\right)^{\prime}\right)_{\bar{E}}=\left(\overline{\left(\mathfrak{\mathcal { A }}_{E}\right)_{b}}\right)^{\prime} .
$$

Proof. We can easily show that $\mathfrak{U}_{E}$ is a symmetric \#-algebra on $E D$. Suppose that $\mathfrak{Y}$ is an $E W^{\sharp}$-algebra. Then we have only to show that $\overline{\left(\mathfrak{A U}_{E}\right)_{b}}$ is a von Neumann algebra. Clearly we have $\left(\overline{\mathfrak{A}}_{b}\right)_{\bar{E}} \subset\left(\overline{\left.\mathfrak{Q}_{E}\right)_{b}}\right.$ and it follows that $\left(\overline{\left.\mathfrak{\mathscr { U }}_{E}\right)_{b}}\right)^{\prime} \subset\left(\left(\overline{\mathfrak{A}}_{b}\right)_{\bar{E}}\right)^{\prime}$. Since $\overline{\mathfrak{A}}_{b}$ is a von Neumann algebra and $\left(\overline{\mathfrak{A}}_{b}\right)^{\prime}=\mathfrak{U}^{\prime}$, we have $\left(\left(\overline{\mathfrak{A}}_{b}\right)_{\bar{E}}\right)^{\prime}=\left(\left(\overline{\mathfrak{A}}_{b}\right)^{\prime}\right)_{\bar{E}}=\left(\mathfrak{Q}^{\prime}\right)_{\bar{E}}$. Next we shall show that $\left(\mathfrak{X}^{\prime}\right)_{\bar{E}} \subset\left(\mathfrak{Q U}_{E}\right)^{\prime}$. Let $C \in \mathfrak{U} \mathfrak{U}^{\prime}$. For each $\xi, \eta \in \mathcal{D}$ and $T \in \mathfrak{A}$ we have

$$
\begin{aligned}
\left(C_{\bar{L}} T_{E} E \xi \mid E \eta\right) & =(C E T E \xi \mid E \eta)=\left(C E \xi \mid(E T E)^{\sharp} E \eta\right) \\
& =\left(C E \xi \mid E T T^{\ddagger} E \eta\right)=\left(C_{\bar{E}} E \xi \mid T_{E}^{\sharp} E \eta\right)
\end{aligned}
$$

and hence $C_{\bar{E}} \in\left(\mathfrak{N}_{E}\right)^{\prime}$, and so $\left(\mathfrak{X}^{\prime}\right)_{\bar{E}} \subset\left(\mathfrak{Q}_{E}\right)^{\prime}$. On the other hand we have $\left(\overline{\left(\mathfrak{A U}_{E}\right)_{b}}\right)^{\prime} \supset\left(\mathfrak{\Re}_{E}\right)^{\prime}$. Therefore we have

$$
\left(\left(\overline{\left.\mathfrak{A}_{E}\right)_{b}}\right)^{\prime} \subset\left(\left(\overline{\mathfrak{A}_{b}}\right)_{\bar{E}}\right)^{\prime}=\left(\overline{\mathfrak{A}_{b}^{\prime}}\right)_{\bar{E}}=\left(\mathfrak{E}^{\prime}\right)_{\bar{E}} \subset\left(\mathfrak{\mathscr { U }}_{E}\right)^{\prime} \subset\left(\overline{\left(\mathfrak{\mathscr { U }}_{E}\right)_{b}}\right)^{\prime}\right.
$$

and it follows that

$$
\left(\left(\overline{\left.\mathfrak{A}_{E}\right)_{b}}\right)^{\prime}=\left(\left(\overline{\mathfrak{A}}_{b}\right)_{\bar{E}}\right)^{\prime}=(\mathfrak{Q})^{\prime}\right)_{\bar{E}}=\left(\mathfrak{U}_{E}\right)^{\prime} .
$$

Therefore we have

$$
\left(\overline{\left(\mathfrak{\Re}_{E}\right)_{b}}\right)^{\prime \prime}=\left(\left(\overline{\mathfrak{A}}_{b}\right)_{\bar{E}}\right)^{\prime \prime}=\left(\overline{\mathfrak{A}_{b}}\right)_{\bar{E}} \subset\left(\overline{\left.\mathfrak{Q}_{E}\right)_{b}} .\right.
$$

Consequently $\mathfrak{U}_{E}$ is an $E W^{\sharp}$-algebra on $E \mathfrak{D}$.

Definition 3.2. Let $\mathfrak{A}$ be an $E W^{\sharp}$-algebra on $\mathfrak{D}$ and let $E \in \mathfrak{Y}_{p}$. 
We call $\mathfrak{A}_{E}$ the reduced $E W^{\sharp}$-algebra of $\mathfrak{A}$.

Proposition 3.3. Let $\mathfrak{A}$ be a closed symmetric \#-algebra on $\mathfrak{D}$ and let $\mathfrak{M}$ be an $\mathfrak{A}$-invariant $\tau_{0}$-closed subspace of $\mathfrak{D}$. Let $A_{\mathfrak{n}}$ be the restriction of $A$ onto $\mathfrak{M}$ and let $\mathfrak{U}_{\mathfrak{m}}=\left\{A_{\mathfrak{m}} ; A \in \mathfrak{A}\right\}$. Then the following conditions are satisfied.

(1) $\mathfrak{U}_{\mathfrak{n}}$ is a closed symmetric \#-algebra on $\mathfrak{M}$ and we have

$$
\mathfrak{M}=\bigcap_{A \in \mathfrak{I}} \mathfrak{D}\left(\overline{A_{\mathfrak{N}}}\right)=\bigcap_{A \in \mathfrak{\mathscr { N }}} \mathfrak{D}\left(A_{\mathfrak{M}}^{*}\right) .
$$

(2) Let $E_{\mathfrak{n}}$ be the projection onto $\overline{\mathfrak{M}}$. Then we have $E_{\mathfrak{m}} \mathfrak{D}=\mathfrak{M}$ and $E_{\mathfrak{n}} \in \mathfrak{U}^{\prime}$.

(3) If $E \in\left(\mathfrak{U}^{\prime}\right)_{p}$, then $E \mathfrak{D}$ is an $\mathfrak{2}$-invariant $\tau_{0}$-closed subspace of $\mathfrak{D}$.

Proof. (1) Under $\left(A_{\mathfrak{n}}\right)^{\sharp}=\left(A^{\sharp}\right)_{\mathfrak{n}}$, clearly $\mathfrak{X}_{\mathfrak{M}}$ is a symmetric \#algebra on $\mathfrak{M}$. By Theorem 2.3 we have $\left(\overline{\left.A_{\mathfrak{n}}\right)^{\sharp}}=\overline{\left(A^{\sharp}\right)_{\mathfrak{M}}}=\left(A_{\mathfrak{n}}\right)^{*}\right.$ for all $A \in \mathfrak{A}$. Furthermore, since $\mathfrak{M}$ is $\tau_{0}$-closed, $\mathfrak{A}_{\mathfrak{n}}$ is closed. Therefore we have

$$
\mathfrak{M}=\bigcap_{A \in \mathfrak{X}} \mathfrak{D}\left(\overline{A_{\mathfrak{N}}}\right)=\bigcap_{A \in \mathfrak{X}} \mathfrak{D}\left(A_{\mathfrak{N}}^{*}\right) .
$$

(2) We shall show $E_{\mathfrak{n}} \mathfrak{D}=\mathfrak{M}$. Clearly we have $\mathfrak{M} \subset E_{\mathfrak{n}} \mathfrak{D}$. Let $\xi \in \mathfrak{D}$. For each $\eta \in \mathfrak{M}$ and $A \in \mathfrak{A}$ we have

$$
\left(A_{\Re} \eta \mid E_{\Re} \xi\right)=\left(E_{\Re} A_{\Re} \eta \mid \xi\right)=(A \eta \mid \xi)=\left(\eta \mid A^{*} \xi\right)
$$

and it follows that $E_{\mathfrak{m}} \xi \in \bigcap_{A \in \mathfrak{r}} \mathfrak{D}\left(A_{\mathfrak{r}}^{*}\right)=\mathfrak{M}$. Consequently we have $\mathfrak{M}=E_{\Re} \mathfrak{D}$. We shall show $E_{\Re} \in \mathfrak{\mathfrak { X } ^ { \prime }}$. For each $A \in \mathfrak{M}$ and $\xi, \eta \in \mathfrak{D}$ we have

$$
\begin{aligned}
\left(E_{\mathfrak{m}} A \xi \mid \eta\right) & =\left(A \xi \mid E_{\mathfrak{n}} \eta\right)=\left(\xi \mid A^{\sharp} E_{\mathfrak{n}} \eta\right)=\left(E_{\mathfrak{m}} \xi \mid A^{\sharp} E_{\mathfrak{n}} \eta\right) \\
& =\left(A E_{\mathfrak{m}} \xi \mid E_{\mathfrak{m}} \eta\right)=\left(E_{\mathfrak{m}} A E_{\mathfrak{m}} \xi \mid \eta\right)=\left(A E_{\mathfrak{m}} \xi \mid \eta\right)
\end{aligned}
$$

and hence $E_{\mathfrak{n}} \in \mathfrak{U}^{\prime}$.

(3) By Proposition 2.8 it is clear that $E \mathfrak{D}$ is an 2 -invariant subspace of $\mathfrak{D}$. We can easily show that $E \mathscr{D}$ is $\tau_{0}$-closed.

DEFINITION 3.4 Let $\mathfrak{A}$ be a closed symmetric \#-algebra on $\mathfrak{D}$ and let $E \in\left(\mathfrak{U}^{\prime}\right)_{p}$. By Proposition $3.3(3), \mathfrak{M}=E \mathfrak{D}$ is an $\mathfrak{U}$-invariant $\tau_{0}$-closed subspace of $\mathfrak{D}$. We define

$$
A_{E}=A_{\mathfrak{R}} \quad \text { and } \quad \mathfrak{A}_{E}=\left\{A_{E} ; A \in \mathfrak{Q}\right\} \text {. }
$$

By Proposition $3.3(1), \mathfrak{A}_{E}$ is a closed symmetric \#-algebra on $\mathfrak{M}$. Clearly the map $A \rightarrow A_{E}$ of $\mathfrak{A}$ onto $\mathfrak{A}_{E}$ is a homomorphism. We call 
this homomorphism the induction of $\mathfrak{U}$ and $\mathfrak{U}_{E}$ is called the induced algebra of $\mathfrak{2}$.

THEOREM 3.5. Let $\mathfrak{A}$ be a closed $E W^{*}$-algebra on $\mathfrak{D}$ and let $E \epsilon$ $\left(\mathfrak{U}^{\prime}\right)_{p}$. Then $\mathfrak{U}_{E}$ is a closed $E W^{\sharp}$-algebra on $E \mathfrak{D}$ and we have $\left(\mathfrak{U}_{E}\right)^{\prime}=$ $\left(\mathfrak{U}^{\prime}\right)_{E}$.

Proof. We shall show that $\left(\overline{\left(\overline{\mathfrak{A}_{E}}\right)_{b}}\right)^{\prime}=\left(\mathfrak{U}^{\prime}\right)_{E}$. Let $C \in\left(\overline{\left(\mathfrak{\mathcal { A }}_{E}\right)_{b}}\right)^{\prime}$, i.e., $C$ is a bounded linear operator on $\overline{E D S}$ such that $C \overline{A_{E}}=A_{E} C$ for every $A_{E} \in\left(\mathfrak{U}_{E}\right)_{b}$. We shall show $C E \in \mathfrak{U}^{\prime}$. For each $A \in \mathfrak{A}$ let $A=$ $U|A|$ be the polar decomposition of $A$. Let $\overline{A \mid}=\int_{0}^{\infty} \lambda d \overline{E_{A}(\lambda)}$ be the spectral decomposition of $\overline{|A|}$. Then we have $U, E_{A}(\lambda) \in \mathfrak{A}_{b}$ for all $\lambda$ and hence $U_{E}, E_{A}(\lambda)_{E} \in\left(\mathfrak{A}_{E}\right)_{b}$. Since $\left.\left(\overline{U_{E}}\right)=(\bar{U})_{E}, \overline{\left(E_{A}(\lambda)_{E}\right.}\right)=\overline{\left(E_{A}(\lambda)\right)_{E}}$ and $C \in\left(\left(\overline{\left.\mathfrak{A}_{E}\right)_{b}}\right)^{\prime}\right.$, we have $C(\bar{U})_{E}=(\bar{U})_{E} C$ and $C\left(\overline{E_{A}(\lambda)}\right)_{E}=\left(\overline{E_{A}(\lambda)}\right)_{E} C$ and hence $C E$ commutes with $\bar{U}$ and $\overline{E_{A}(\lambda)}$. Therefore $C E$ commutes with $\bar{A}$. Then, clearly we have $C E \in \mathfrak{U}^{\prime}$ and hence $C=(C E)_{E} \in\left(\mathfrak{U}^{\prime}\right)_{E}$. Therefore we get $\left(\left(\overline{\left.\mathfrak{A}_{E}\right)_{b}}\right)^{\prime} \subset\left(\mathfrak{Q}^{\prime}\right)_{E}\right.$. Conversely we can easily show $\left(\left(\overline{\left.\mathfrak{A}_{E}\right)_{b}}\right)^{\prime} \supset\left(\mathfrak{Q}^{\prime}\right)_{E}\right.$. Consequently we have $\left(\left(\overline{\left.\mathfrak{A}_{E}\right)_{b}}\right)^{\prime}=\left(\mathfrak{Y}^{\prime}\right)_{E}\right.$. We shall show $\left(\overline{\left(\mathfrak{Q}_{E}\right)_{b}}\right)^{\prime \prime}=\overline{\left(\mathfrak{A}_{E}\right)_{b}}$. By the above argument, ([3] Ch. I, §2, Prop. 1) and Proposition 2.9 we have

$$
\left(\overline{\left(\mathfrak{\Re}_{E}\right)_{b}}\right)^{\prime \prime}=\left(\left(\mathfrak{U}^{\prime}\right)_{E}\right)^{\prime}=\left(\mathfrak{U}^{\prime \prime}\right)_{E}=\left(\overline{\mathfrak{A}}_{b}\right)_{E} .
$$

On the other hand, clearly we have $\left.\left(\overline{\mathfrak{A}_{b}}\right)_{E} \subset \overline{\left(\mathfrak{A}_{E}\right.}\right)_{b}$ and hence $\left(\overline{\left(\mathfrak{P}_{E}\right)_{b}}\right)^{\prime \prime}=$ $\left(\overline{\mathfrak{A}_{b}}\right)_{E} \subset\left(\overline{\left.\mathfrak{A}_{E}\right)_{b}}\right.$ and it follows that $\left(\left(\overline{\left.\mathfrak{A}_{E}\right)_{b}}\right)^{\prime \prime}=\overline{\left(\mathfrak{\Re}_{E}\right)_{b}}\right.$. Consequently $\mathfrak{X}_{E}$ is an $E W^{\sharp}$-algebra on $E \mathfrak{D}$. Furthermore we have $\left(\mathfrak{U}_{E}\right)^{\prime}=\left(\overline{\left(\mathfrak{\mathscr { V }}_{E}\right)_{b}}\right)^{\prime}=$ $\left(\mathfrak{X}^{\prime}\right)_{E}$, by Proposition 2.9.

DeFINITION 3.6. Let $\mathfrak{U}$ be a closed $E W^{\sharp}$-algebra on $\mathfrak{D}$ and let $E \in\left(\mathfrak{U}^{\prime}\right)_{p}$. Then $\mathfrak{N}_{E}$ is called the induced $E W^{\sharp}$-algebra of $\mathfrak{i}$.

Next we shall study the product of $E W^{\sharp}$-algebras. Let $\left\{\mathfrak{P}, \mathfrak{D}_{\ell}\right\}_{\in A}$ be a family of symmetric $\#$-algebras $\mathfrak{U}_{c}$ on $\mathfrak{D}_{\imath}$. Let $\mathfrak{S}_{2}$, be the completion of $\mathfrak{D}_{\text {, for each }} \iota \in \Lambda$ and let $\mathfrak{S}$ be the direct sum of $\left\{\mathfrak{S}_{\ell}\right\}_{\in \in A}$. We denote the product of $\left\{\mathfrak{I}_{\iota}\right\}_{\epsilon \in A}$ by $\mathfrak{U}=\Pi_{\epsilon \in A} \mathfrak{A}$, and define $\mathfrak{X}$ as follows. Let

$$
\begin{aligned}
\mathfrak{D}(\mathfrak{U})= & \left\{\left(\xi_{\iota}\right)_{\iota \in \Lambda} \in \mathfrak{S} ; \xi_{\iota} \in \mathfrak{D}, \text { for all } \iota \in A\right. \text { and } \\
& \left.\sum_{\iota \in A}\left\|A_{\iota} \xi_{\iota}\right\|^{2}<\infty \text { for all } A_{\iota} \in \mathfrak{U}\right\} .
\end{aligned}
$$

We define

$$
A \xi=\left(A_{\iota}\right)_{\iota \in \Lambda}\left(\xi_{\iota}\right)_{\iota \in \Lambda}=\left(A_{\iota} \xi_{\iota}\right)_{\iota \in \Lambda}
$$

for all $\xi=\left(\xi_{\iota}\right)_{\iota \in \Lambda} \in \mathfrak{D}(\mathfrak{U})$ and $A=\left(A_{\iota}\right)_{\iota \in \Lambda} \in \mathfrak{X}$. It is clear that $\prod_{\iota \in \Lambda} \mathfrak{A}$, 
is a \#-algebra on $\mathfrak{D}(\mathfrak{U})$ under the following operations; $A+B=$ $\left(A_{\iota}+B_{\iota}\right)_{\iota \in \Lambda}, \lambda A=\left(\lambda A_{\iota}\right)_{\iota \in \Lambda}, A B=\left(A_{\iota} B_{\iota}\right)_{\iota \in \Lambda}, A^{\sharp}=\left(A_{\iota}^{\ddagger}\right)$, for each $A=$ $\left(A_{\iota}\right)_{\iota \in A}, B=\left(B_{\iota}\right)_{\iota \in A} \in \prod_{\iota \in A} \mathfrak{A} \iota$ and $\lambda \in \mathfrak{E}$.

THEOREM 3.7. Let $\left\{\mathfrak{A}_{\ell}\right\}_{\in A}$ be a family of (resp. closed) symmetric \#-algebras $\mathfrak{A}_{\epsilon}$ on $\mathfrak{D}_{\iota}$. Then $\mathfrak{A}=\Pi_{\iota \in \Lambda} \mathfrak{A}_{\epsilon}$ is a (resp. closed) symmetric \#-algebra on $\mathfrak{D}(\mathfrak{A})$. In particular, if $\mathfrak{U}_{c}$ is an $E W^{\sharp}$-algebra on $\mathfrak{D}$, for every $\iota \in \Lambda$, then $\mathfrak{A}$ is an $E W^{\#-a l g e b r a ~ o n ~} \mathfrak{D}(\mathfrak{A})$ and we have

$$
\mathfrak{U} \mathfrak{U}^{\prime}=\bigoplus_{\iota \in A} \mathfrak{U}_{\iota}^{\prime} \quad \text { and } \quad \overline{\mathfrak{A}}_{b}=\bigoplus_{\iota \in A}\left(\overline{\left.\mathfrak{A}_{\iota}\right)_{b}},\right.
$$

where we denote by $\bigoplus_{\llcorner\in A} \mathfrak{B}_{\iota}$ the direct sum of a family $\left\{\mathfrak{B}_{\iota}\right\}_{\iota \in \Lambda}$ of von Neumann algebras.

Proof. If $\mathfrak{A}$, is a (resp. closed) symmetric $\#$-algebra on $\mathfrak{D}_{\iota}$ for all $\iota \in \Lambda$, it is easily shown that $\Pi_{\iota \in \Lambda} \mathfrak{A}$, is a (resp. closed) symmetric \#-algebra on $\mathfrak{D}(\mathfrak{U})$. We shall show that $\overline{\mathfrak{A}}_{b}=\bigoplus_{\iota \in A}\left(\overline{\left.\mathfrak{A}_{t}\right)_{b}}\right.$, if $\mathfrak{A}_{t}$ is an $E W^{\sharp}$-algebra on $\mathfrak{D}_{\iota}$ for every $\iota \in \Lambda$. Suppose that $A=\left(A_{\iota}\right)_{\iota \in \Lambda} \in \mathfrak{U}_{b}$. We can easily show that $A_{\iota} \in\left(\mathfrak{H}_{\iota}\right)_{b}$ for every $\iota \in \Lambda$ and $\sup _{\iota \in A}\left\|\bar{A}_{\iota}\right\| \leqq$ $\|\bar{A}\|$. For each $\xi=\left(\xi_{\iota}\right)_{\iota \in A} \in \mathfrak{D}(\mathfrak{U})$ we have $A \xi=\left(\bar{A}_{\iota}\right)_{\iota \in \Lambda} \xi$ and hence $\bar{A}=\left(\bar{A}_{\iota}\right)_{\iota \in \Lambda}$, and so $\bar{A} \in \bigoplus_{\iota \in \Lambda}\left({\overline{\mathfrak{A}} \iota)_{b}}_{b}\right.$. Conversely suppose $X=\left(X_{\iota}\right)_{\iota \in \Lambda} \in$ $\bigoplus_{\iota \in A}\left(\overline{\left.\hat{A}_{c}\right)_{b}}\right.$. Then there is an element $A_{\iota}$ in $\left(\mathfrak{A}_{\iota}\right)_{b}$ such that $X_{\iota}=\bar{A}_{c}$ for all $\iota \in \Lambda$. Let $A=\left(A_{\iota}\right)_{\iota \in \Lambda}$. We can easily show that $A \in \mathfrak{A}_{b}$ and $\bar{A}=\left(\bar{A}_{\imath}\right)_{\iota \in A}=X$. Therefore we have $X \in \overline{\mathfrak{A}}_{b}$. Consequently we have $\overline{\mathfrak{A}}_{b}=\bigoplus_{\epsilon \in A}\left(\overline{\left.\mathfrak{2})_{c}\right)_{b}}\right.$. Since $\bigoplus_{\epsilon \in A}\left(\overline{\left.\mathfrak{A}_{\iota}\right)_{b}}\right.$ is a von Neumann algebra, $\mathfrak{A}$ is an $E W^{\sharp}$-algebra on $\mathfrak{D}(\mathfrak{Q})$. Furthermore we have $\mathfrak{Q}^{\prime}=\left(\overline{\mathfrak{A}}_{b}\right)^{\prime}=\left(\bigoplus_{t \in A}(\overline{\mathfrak{2}})_{b}\right)^{\prime}=$ $\bigoplus_{\imath \in I}\left(\left(\overline{\left.\mathfrak{A}_{c}\right)_{b}}\right)^{\prime}=\bigoplus_{\iota \in A} \mathfrak{A}^{\prime}\right.$, by Proposition 2.9.

Definition 3.8. Let $\mathfrak{2}($ resp. $\mathfrak{B})$ be a symmetric \#-algebra on $\mathfrak{D}$ (resp. (5)). The map. $\Phi$ of $\mathfrak{A}$ into $\mathfrak{B}$ is called a homomorphism if it is linear, if $\Phi(S T)=\Phi(S) \Phi(T), S, T \in \mathfrak{A}$, and if $\Phi\left(S^{\sharp}\right)=\Phi(S)^{\sharp}, S \in \mathfrak{A}$. If $\Phi$ is a bijective homomorphism of $\mathfrak{A}$ onto $\mathfrak{B}$, then it is called an isomorphism of $\mathfrak{X}$ onto $\mathfrak{B}$. Then $\mathfrak{A}$ and $\mathfrak{B}$ are called isomorphic. Let $\Phi$ be an isomorphism of $\mathfrak{A}$ onto $\mathfrak{B}$. If there is an isometric mapping $U$ of $\mathfrak{D}$ onto $\mathbb{S}$ such that $\Phi(S)=U S U^{-1}$ for every $S \in \mathfrak{A}$, then $\Phi$ is called a spatial isomorphism and we call $\mathfrak{A}$ and $\mathfrak{B}$ are spatial isomorphic and write by $\mathfrak{U} \underset{\overline{(s)}}{\cong} \mathfrak{B}$.

Proposition 3.9. Let $\mathfrak{2}$ be a closed $E W^{\sharp}$-algebra on $\mathfrak{D}$ and let $\left\{E_{c}\right\}_{\in A}$ be a family of mutually orthogonal projections in $\mathfrak{X}^{\prime}$ such

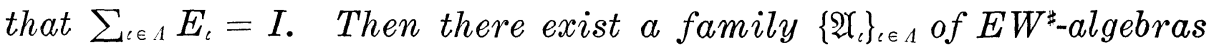
and a spatial isomorphism $\Phi$ of $\mathfrak{A}$ onto the $E W^{\sharp}$-subalgebra of $\Pi_{\iota \in 1} \mathfrak{A}$, such that $\overline{\Phi(\mathfrak{A})_{b}}=\bigoplus_{\iota \in \Lambda} \overline{(\mathfrak{A})_{b}}$. 
Proof. Let $\mathfrak{D}_{c}=E_{\imath}\left(\mathfrak{D}\right.$ and let $\mathscr{S}_{c}$ be the completion of $\mathfrak{D}_{\iota}$. Then $\mathfrak{A}_{\imath}=\mathfrak{A}_{E_{\ell}}$ is a closed $E W^{\sharp}$-algebra on $\mathfrak{D}_{\iota}$, by Theorem 3.5. It is easy to show that $\Phi ; A \rightarrow\left(A_{\iota}\right)_{\epsilon \in \Lambda}\left(A_{\iota}=A_{E_{\ell}}\right)$ is an isomorphism of $\mathfrak{A}$ into $\Pi_{\iota \in A} \mathfrak{A} \iota$. We define the mapping $U$ of $\mathfrak{D}$ into $\bigoplus_{\iota \in \Lambda} \mathfrak{F}_{\iota}$ by $U \xi=\left(E_{\iota} \xi\right)_{\iota \in A}$. Then $U$ is an isometric mapping of $\mathfrak{D}$ onto $\mathscr{D} \Phi(\mathfrak{U})$. In fact, let $\xi \in \mathfrak{D}$ and then $\xi_{\iota}=E_{\iota} \xi \in \mathfrak{D}_{\iota}$ for all $\iota \in \Lambda$ and we have, for each $A_{\iota} \in \mathfrak{A}$,

$$
\sum_{\iota \in, I}\left\|A_{\iota} \xi_{\iota}\right\|^{2}=\sum_{\iota \in, l}\left\|A E_{\iota} \xi\right\|^{2}=\sum_{\iota \in, A}\left\|E_{\iota} A \xi\right\|^{2}=\|A \xi\|^{2}<\infty
$$

and hence $\left(E_{\iota} \xi\right)_{\iota \in \Lambda} \in \mathfrak{D} \Phi(\mathfrak{Q})$. Conversely suppose that $\left(\xi_{\iota}\right)_{\iota \in \Lambda} \in \mathfrak{D}\left(\Pi_{\iota \in \Lambda} \mathfrak{U}\right)$, i.e., $\xi_{\iota} \in \mathfrak{D}_{\iota}=E_{\iota} \mathfrak{D}$ and $\sum_{\iota \in A}\left\|A_{\iota} \xi_{\iota}\right\|^{2}<\infty$ for all $A \in \mathfrak{A}$. Let $\xi=\sum_{\iota \in A} \xi_{\iota}$. Then we have

$$
\sum_{\iota \in A}\left\|A \xi_{\iota}\right\|^{2}=\sum_{\iota \in A}\left\|A E_{\iota} \xi\right\|^{2}=\sum_{\iota \in, A}\left\|A_{\iota} \xi_{\iota}\right\|^{2}<\infty
$$

for all $A \in \mathfrak{A}$ and therefore $\xi \in \mathfrak{D}(\bar{A})$ for all $A \in \mathfrak{A}$. Since $\mathfrak{U}$ is closed, we have $\xi \in \mathfrak{D}$ and $U \xi=\left(\xi_{\varepsilon}\right)_{\epsilon \in \Lambda}$. Consequently $U$ is onto. Furthermore we have

$$
\|U \xi\|^{2}=\left\|\left(E_{\iota} \xi\right)_{\iota \in A}\right\|^{2}=\sum_{i \in A}\left\|E_{\iota} \xi\right\|^{2}=\|\xi\|^{2}
$$

and hence $\bar{U}$ is an isometric mapping of $\mathscr{K}$ onto $\bigoplus_{\iota \in A} \mathscr{H}_{\iota}$. Finally we shall show that $U A U^{-1}=\left(A_{\iota}\right)_{\iota \in \Lambda}$ for all $A \in \mathfrak{A}$. For each $\xi \in \mathfrak{D}$ we have

$$
U A U^{-1}\left(E_{\iota} \xi\right)_{\iota \in \Lambda}=U A \xi=\left(E_{\iota} A \xi\right)_{\iota \in \Lambda}=\left(A E_{\iota} \xi\right)_{\iota \in \Lambda}=\left(A_{\iota} E_{\iota} \xi\right)_{\iota \in \Lambda}
$$

and

$$
\left(A_{\iota}\right)_{\iota \in \Lambda}\left(E_{\iota} \xi\right)_{\iota \in \Lambda}=\left(A_{\iota} E_{\iota} \xi\right)_{\iota \in \Lambda}
$$

and hence $U A U^{-1}=\left(A_{\imath}\right)_{\iota \in \Lambda}$. By ([3] Ch. I, $\left.\S 2,2\right)$ it is easy to show that $\overline{\Phi(\mathfrak{A})_{b}}=\bigoplus_{\iota \in A} \overline{(\mathfrak{A})_{c}}$. Consequently $\Phi(\mathfrak{U})$ is an $E W^{\sharp}$-subalgebra of $\Pi_{\iota \in A} \mathfrak{\mathfrak { U } _ { \iota }}$ with $\overline{\Phi(\mathfrak{U})_{b}}=\bigoplus_{\iota \in A} \overline{(\mathfrak{N} \iota)_{b}}$.

Proposition 3.10. Let $\mathfrak{N}$, be a closed $E W^{\sharp}$-algebra on $\mathfrak{D}$, for all $\iota \in \Lambda$ and let $\mathfrak{A}=\prod_{\iota \in \Lambda} \mathfrak{A}$. If $F_{\iota} \in\left(\mathfrak{U}_{c}^{\prime}\right)_{p}$ for every $\iota \in \Lambda$, then $F=$ $\left(F_{c}\right)_{\iota \in \Lambda} \in\left(\mathfrak{V}^{\prime}\right)_{p}$ and furthermore we have

$$
\mathfrak{A}_{F}=\prod_{\iota \in A}\left(\mathfrak{A}_{\iota}\right)_{F_{\iota}} \text { and }\left(\mathfrak{A}_{F}\right)^{\prime}=\bigoplus_{\iota \in A}\left(\mathfrak{U}_{\iota}^{\prime}\right)_{F_{\iota}} .
$$

Proof. Clearly $F=\left(F_{\iota}\right)_{\iota \in \Lambda} \in\left(\mathfrak{U}^{\prime}\right)_{p} . \quad$ Let $\mathfrak{B}=\prod_{\iota \in \Lambda}\left(\mathfrak{U}_{\iota}\right)_{F_{\ell}}$. Then we have

$$
\mathfrak{D}\left(\mathfrak{U}_{F}\right)=F \mathfrak{D}(\mathfrak{U})=\left\{\left(F_{\iota} \xi_{\iota}\right)_{\iota \in \Lambda} ; \xi=\left(\xi_{\iota}\right)_{\iota \in \Lambda} \in \mathfrak{D}(\mathfrak{U})\right\}
$$

and 


$$
\begin{aligned}
\mathfrak{D}(\mathfrak{B})= & \left\{\left(F_{\iota} \xi_{\iota}\right)_{\iota \in \Lambda} ; \xi_{\iota} \in \mathfrak{D}_{\iota} \text { for all } \iota \in \Lambda\right. \text { and } \\
& \left.\sum_{\iota \in \Lambda}\left\|\left(A_{\iota}\right)_{F_{\iota}} F_{\iota} \xi_{\iota}\right\|^{2}<\infty \text { for all } A_{\iota} \in \mathfrak{A}_{\iota}\right\},
\end{aligned}
$$

and so it is easy to show that $\mathfrak{D}\left(\mathfrak{A}_{F}\right)=\mathfrak{D}(\mathfrak{B})$. Consequently we have $\mathfrak{A}_{F}=\Pi_{\iota \in \Lambda}\left(\mathfrak{A}_{\iota}\right)_{F_{\iota}} \cdot \quad$ By Theorem 3.5 and Theorem 3.7 we have

$$
\left(\mathfrak{U}_{F}\right)^{\prime}=\left(\prod_{\iota \in \Lambda}\left(\mathfrak{U}_{\iota}\right)_{F_{\iota}}\right)^{\prime}=\bigoplus_{\iota \in \Lambda}\left(\left(\mathfrak{U}_{\iota}\right)_{F_{\iota}}\right)^{\prime}=\bigoplus_{\iota \in \Lambda}\left(\mathfrak{U}_{\iota}^{\prime}\right)_{F_{\iota}} .
$$

We define the amplification of an $E W^{\sharp}$-algebra $\mathfrak{A}$ onto $\mathfrak{A} \widetilde{\otimes} I$. Let $\mathfrak{N}_{1}$ and $\mathfrak{N}_{2}$ be $E W^{\sharp}$-algebras on $\mathfrak{D}_{1}$ and $\mathfrak{D}_{2}$ respectively. Let $\mathfrak{D}$ be the subspace of $\mathfrak{S}_{\mathcal{L}}=\mathfrak{S}_{1} \otimes \mathfrak{F}_{2}$ generated by $\left\{\xi_{1} \otimes \xi_{2} ; \xi_{1} \in \mathfrak{D}_{1}, \xi_{2} \in \mathfrak{D}_{2}\right\}$ and denoted by $\mathfrak{D}_{1} \otimes \mathfrak{D}_{2}$. Clearly $\mathfrak{D}$ is a dense subspace of $\mathfrak{S}_{\mathrm{C}}$. For each $T_{1} \in \mathfrak{A}_{1}$ and $T_{2} \in \mathfrak{A}_{2}$ we get an operator $T_{1} \otimes T_{2}$ on $\mathfrak{D}$ defined by $\left(T_{1} \otimes T_{2}\right)\left(\xi_{1} \otimes \xi_{2}\right)=\left(T_{1} \xi_{1}\right) \otimes\left(T_{2} \xi_{2}\right)$, for each $\xi_{1} \in \mathscr{D}_{1}$ and $\xi_{2} \in \mathfrak{D}_{2}$ Then we have, for each $T_{1}, S_{1} \in \mathfrak{N}_{1}$ and $T_{2}, S_{2} \in \mathfrak{A}_{2}, T_{1} \otimes T_{2}$ is a bilinear function of $T_{1}$ and $T_{2} ;\left(T_{1} \otimes T_{2}\right)\left(S_{1} \otimes S_{2}\right)=T_{1} S_{1} \otimes T_{2} S_{2} ;\left(T_{1} \otimes T_{2}\right)^{\#}=$ $T_{1}^{\#} \otimes T_{2}^{\#}$. Then the following proposition is easily shown.

Proposition 3.11. Let $\mathfrak{N}_{1}$ be an $E W^{\sharp}$-algebra on $\mathfrak{D}_{1}$ and let $\mathfrak{S}_{2}$ be a Hilbert space. Putting

$$
\mathfrak{N}_{1} \otimes I_{\mathfrak{S}_{2}}=\left\{T_{1} \otimes I_{H_{2}} ; T_{1} \in \mathfrak{P}_{1}\right\},
$$

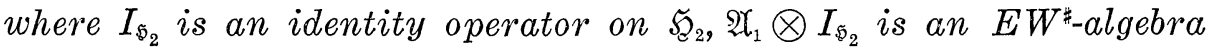
on $\mathfrak{D}_{1} \otimes \mathfrak{S}_{2}$ and we have

$$
\overline{\left(\mathfrak{\mathcal { V }}_{1} \otimes I_{\mathfrak{\xi}_{2}}\right)_{b}}=\left(\overline{\left.\mathfrak{\mathcal { V }}_{1}\right)_{b}} \otimes I_{\mathfrak{S}_{2}} .\right.
$$

Putting

$\mathfrak{D}_{1} \widetilde{\otimes}{\mathscr{\mathfrak { F } _ { 2 }}}_{2}=\bigcap_{T_{1} \in \mathfrak{I}_{1}} \mathfrak{D}\left(\overline{T_{1} \otimes I_{\mathfrak{F}_{2}}}\right),\left(T_{1} \widetilde{\otimes} I_{\mathfrak{F}_{2}}\right) x=\overline{T_{1} \otimes I_{\mathfrak{F}_{2}}} x, x \in \mathfrak{D}_{1} \widetilde{\otimes} \mathfrak{S}_{2}$, $\mathfrak{N}_{1} \widetilde{\otimes} I_{\mathfrak{S}_{2}}=\left\{T_{1} \widetilde{\otimes} I_{\mathfrak{\$}_{2}} ; T_{1} \in \mathfrak{N}_{1}\right\}$ is the closure of $\mathfrak{N}_{1} \otimes I_{\mathfrak{\$}_{2}}$, and so $\mathfrak{N}_{1} \widetilde{\otimes} I_{\mathfrak{\$}_{2}}$ is a closed $E W^{\sharp}$-algebra on $\mathfrak{D}_{1} \widetilde{\otimes} \mathfrak{S}_{2}$.

Definition 3.12. The isomorphism; $T_{1} \rightarrow T_{1} \widetilde{\otimes} I_{\mathfrak{y}_{2}}$ is called an amplification of $\mathfrak{U}_{1}$ onto $\mathfrak{U}_{1} \widetilde{\otimes} I_{\mathfrak{F}_{2}}$.

4. Preduals of $E W^{\sharp}$-algebras. Let $\mathfrak{M}$ be a symmetric $\#$-algebra on $\mathfrak{D}$. Let $\mathfrak{S}_{\infty}=\bigoplus_{n=1}^{\infty} \mathfrak{S}_{n}$, where $\mathfrak{S}_{n}$ is the replica of $\mathfrak{S}_{2}$ for $n=1$, $2, \cdots$. For each $\xi=\left(\xi_{1}, \xi_{2}, \cdots, \xi_{n}, \cdots\right) \in \mathfrak{D}_{\infty}(\mathfrak{U})$ and $T \in \mathfrak{N}$, putting $T_{\infty} \xi_{\infty}=\left(T \xi_{1}, T \xi_{2}, \cdots, T \xi_{n}, \cdots\right)$, we get a linear operator $T_{\infty}$ on $\mathfrak{D}_{\infty}(\mathfrak{A})$. Let $\mathfrak{N}_{\infty}=\left\{T_{\infty} ; T \in \mathfrak{X}\right\}$. Then we have, for each $S$ and $T$ in $\mathfrak{X}, T_{\infty}+$ $S_{\infty}=(T+S)_{\infty}, \lambda T_{\infty}=(\lambda T)_{\infty}, T_{\infty} S_{\infty}=(T S)_{\infty}, T_{\infty}^{\#}=\left(T^{\sharp}\right)_{\infty}$, and so the following lemma is easily shown. 
Lemma 4.1. Let $\mathfrak{A}$ be a (resp. closed) symmetric \#-algebra on D. Then $\mathfrak{N}_{\infty}$ is a (resp. closed) symmetric \#-algebra on $\mathfrak{D}_{\infty}(\mathfrak{U})$.

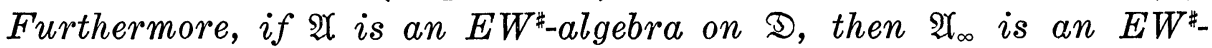
algebra on $\mathfrak{D}_{\infty}(\mathfrak{U})$.

Let $\mathfrak{A}$ be a symmetric \#-algebra on $\mathfrak{D}$. A linear functional $\varphi$ on $\mathfrak{A}$ is called positive if $\varphi\left(A^{\sharp} A\right) \geqq 0$ for every $A \in \mathfrak{N}$ and we denote by $\varphi \geqq 0$.

For each $\xi \in \mathfrak{D}$ and $y \in \mathfrak{S}$, putting

$$
\omega_{\xi, y}(T)=(T \xi \mid y), \quad T \in \mathfrak{Q},
$$

$\omega_{\xi, y}$ is a strongly continuous linear functional on $\mathfrak{N}$. In particular, we denote $\omega_{\xi, \xi}(\xi \in \mathcal{D})$ by $\omega_{\xi}$.

Lemia 4.2. Let $\mathfrak{A}$ be a closed symmetric \#-algebra on D. Suppose that $\varphi$ is a positive linear functional on $\mathfrak{A}$ and $\xi \in \mathfrak{D}$. If $\varphi \leqq \omega_{\xi}$, then there exists $a C \in \mathfrak{X}^{\prime}$ such that $0 \leqq C \leqq I$ and $\varphi=\omega_{C \xi}$.

Proof. For each $S, T \in \mathfrak{A}$ we have

$$
\left|\varphi\left(S^{\sharp} T\right)\right|^{2} \leqq \varphi\left(S^{\sharp} S\right) \varphi\left(T^{\sharp} T\right) \leqq\|S \xi\|^{2}\|T \xi\|^{2} .
$$

Putting $B(T \xi, S \xi)=\varphi\left(S^{\sharp} T\right), B($, ) is an hermitian positive sesquilinear form on $\mathfrak{A} \xi$ with norm $\leqq 1$, so that there is an hermitian positive operator $C_{0}$ in $\mathscr{B} \overline{(\mathfrak{A} \xi)}$ such that $\left\|C_{0}\right\| \leqq 1$ and for all $S$ and $T$ in $\mathfrak{A}$ $\varphi\left(S^{\sharp} T\right)=\left(T \xi \mid C_{0} S \xi\right)$. Since $\mathfrak{A} \xi$ is an $\mathscr{X}$-invariant subspace of $\mathfrak{D}$, the projection $E_{\xi}$ onto $\overline{\mathfrak{A} \xi}$ belongs to $\mathfrak{C}^{\prime}$ (Proposition 3.3). Putting $C^{\prime}=$ $C_{0} E_{\xi}$, for each $A, B$ and $T$ in $\mathfrak{U}$ we have

$$
\begin{aligned}
\left(T C^{\prime} A \xi \mid B \xi\right) & =\left(T C_{0} E_{\xi} A \xi \mid B \xi\right)=\left(T C_{0} A \xi \mid B \xi\right)=\left(A \xi \mid C_{0} T^{\sharp} B \xi\right) \\
& =\varphi\left(\left(T^{\sharp} B\right)^{\sharp} A\right)=\varphi\left(B^{\sharp} T A\right)=\left(T A \xi \mid C_{0} B \xi\right) \\
& =\left(C_{0} T A \xi \mid B \xi\right)=\left(C^{\prime} T A \xi \mid B \xi\right)
\end{aligned}
$$

and since $\mathfrak{A} \xi$ is dense in $E_{\xi} \mathfrak{D}$ under the induced topology $\tau_{0}$, we get

$$
\left(T C^{\prime} E_{\xi} \xi_{1} \mid E_{\xi} \eta_{1}\right)=\left(C^{\prime} T E_{\xi} \xi_{1} \mid E_{\xi} \eta_{1}\right)
$$

for every $\xi_{1}, \eta_{1} \in \mathfrak{D}$ and furthermore we have

$$
\left(T C^{\prime}\left(I-E_{\xi}\right) \xi_{1} \mid \eta_{1}\right)=0=\left(C_{0} T E_{\xi}\left(I-E_{\xi}\right) \xi_{1} \mid \eta_{1}\right)=\left(C^{\prime} T\left(I-E_{\xi}\right) \xi_{1} \mid \eta_{1}\right) .
$$

Hence we have $\left(T C^{\prime} \xi_{1} \mid \eta_{1}\right)=\left(C^{\prime} T \xi_{1} \mid \eta_{1}\right)$ for every $T \in \mathfrak{X}$ and $\xi_{1}, \eta_{1} \in \mathfrak{D}$. Consequently we get $C^{\prime} \in \mathfrak{2} \mathfrak{X}^{\prime}$ and clearly $C^{\prime}$ is an hermitian positive operator and $\left\|C^{\prime}\right\| \leqq 1$. Now, putting $C=C^{1 / 2}$, for all $T \in \mathfrak{A}$,

$$
\varphi(T)=\left(T \xi \mid C^{\prime} \xi\right)=\omega_{C \xi}(T) \text {. }
$$

Proposition 4.3. Let $\mathfrak{A}$ be a closed symmetric $\#$-algebra on $\mathfrak{D}$ 
and let $\varphi$ be a positive linear functional on $\mathfrak{A}$. Then

(I) the following conditions are equivalent;

(1) $\varnothing$ is weakly continuous;

(2) $\varphi=\sum_{i=1}^{n} \omega_{\xi_{i}}, \xi_{i} \in \mathfrak{D}, i=1,2, \cdots, n$;

(II) the following conditions are equivalent;

(3) $\varphi$ is $\sigma$-weakly continuous;

(4) $\varphi=\sum_{n=1}^{\infty} \omega_{\xi_{n}}, \xi_{\infty}=\left(\xi_{1}, \xi_{2}, \cdots, \xi_{n}, \cdots\right) \in \mathfrak{D}_{\infty}(\mathfrak{U})$.

Proof. $\quad(2) \Rightarrow(1)$ and $(4) \Rightarrow(3)$; clear.

$(3) \Rightarrow(4)$; By Lemma 4.1. $\mathfrak{U}_{\infty}$ is a closed symmetric \#-algebra on $\mathfrak{D}_{\infty}(\mathfrak{A})$. Putting $\varphi_{\infty}\left(T_{\infty}\right)=\varphi(T), T \in \mathfrak{A}, \varphi_{\infty}$ is a positive linear functional on $\mathfrak{U}_{\infty}$. Furthermore, since $\varphi$ is $\sigma$-weakly continuous, there is an $\eta_{\infty}=\left(\eta_{1}, \eta_{2}, \cdots, \eta_{n}, \cdots\right)$ in $\mathfrak{D}_{\infty}(\mathfrak{U})$ such that

$$
\left|\varphi_{\infty}\left(T_{\infty}\right)\right|=|\varphi(T)| \leqq\left|\sum_{n=1}^{\infty}\left(T \eta_{n} \mid \eta_{n}\right)\right|=\left|\left(T_{\infty} \eta_{\infty} \mid \eta_{\infty}\right)\right| .
$$

Hence $\varphi_{\infty}$ is a positive linear functional on $\mathfrak{A}_{\infty}$ and $\varphi_{\infty} \leqq \omega_{\eta_{\infty}}$. By Lemma 4.2. there is a $\xi_{\infty}=\left(\xi_{1}, \xi_{2}, \cdots, \xi_{n}, \cdots\right)$ in $\mathfrak{D}_{\infty}(\mathfrak{U})$ such that $\varphi_{\infty}=\omega_{\xi \infty}$. For each $T \in \mathfrak{A}$ we have

$$
\varphi(T)=\varphi_{\infty}\left(T_{\infty}\right)=\omega_{\xi_{\infty}}(T)=\sum_{n=1}^{\infty}\left(T \xi_{n} \mid \xi_{n}\right)=\sum_{n=1}^{\infty} \omega_{\xi_{n}}(T) .
$$

$(1) \Rightarrow(2)$; By a slight modification of the argument $(3) \Rightarrow(4)$ we can easily show $(1) \Rightarrow(2)$.

Definition 4.4. We denote by $\mathfrak{U}_{*}\left(\right.$ resp. $\left.\mathfrak{U}_{*}^{+}\right)$the set of all $\sigma$ weakly continuous (resp. positive) linear functionals on $\mathfrak{U}$ and $\mathfrak{U}_{*}$ is called the predual of $\mathfrak{A}$.

For $A \in \mathfrak{X}$ and $f \in \mathfrak{U}_{*}$, we define actions of $\mathfrak{U}$ on $f$ by;

$$
(f A)(T)=f(A T),(A f)(T)=f(T A)
$$

for each $T \in \mathfrak{Z}$. Then we have $f A, A f \in \mathfrak{A}_{*}$.

Let $\mathfrak{A}$ be a closed $E W^{\sharp}$-algebra on $\mathfrak{D}$. By Lemma 4.1. $\mathfrak{A}_{\infty}$ is a closed $E W^{\sharp}$-algebra on $\mathfrak{D}_{\infty}(\mathfrak{U})$. For each $T \in \mathfrak{A}$ and $\varphi \in \mathfrak{U}_{*}\left(\right.$ resp. $\mathfrak{X}_{*}^{+}$) putting $\varphi_{\infty}\left(T_{\infty}\right)=\varphi(T), \varphi_{\infty}$ is a weakly continuous (resp. positive) linear functional on $\mathfrak{U}_{\infty}$. Moreover, for each $T \in \mathfrak{A}_{b}$ and $\varphi \in \mathfrak{U}_{*}\left(\right.$ resp. $\left.\mathfrak{U}_{*}^{+}\right)$ putting $\bar{\varphi}(\bar{T})=\varphi(T), \bar{\varphi}$ belongs to the predual $\left(\overline{\mathfrak{A}}_{b}\right)_{*}\left(\operatorname{resp} .\left(\overline{\mathfrak{A}}_{b}\right)_{*}^{+}\right)$of a von Neumann algebra $\overline{\mathfrak{A}}_{b}$.

Lemma 4.5. Let $\mathfrak{A}$ be a closed $E W^{\sharp}$-algebra on $\mathfrak{D}$. Let $\varphi$ and $\psi$ in $\mathfrak{A}_{*}$.

(1) If $\bar{\varphi}=\bar{\psi}$, then $\varphi=\psi$.

(2) If $\bar{\varphi} \geqq 0$, then $\varphi \geqq 0$. 
Proof. (1) For each $T \in \mathfrak{A}$, let $T_{\infty}=U_{\infty}\left|T_{\infty}\right|$ be the polar decomposition of $T_{\infty}$. Then we have $U_{\infty} \in\left(\mathscr{H}_{\infty}\right)_{b}$ and $\left|T_{\infty}\right| \in\left(\mathfrak{H}_{\infty}\right)_{h}^{+}$. Let $\left|T_{\infty}\right|=\int_{0}^{\infty} \lambda d E(\lambda)$ be the spectral decomposition of $\left|\overline{T_{\infty}}\right|$ and for each $n$, putting $\bar{X}_{n}=\int_{0}^{n} \lambda d E(\lambda)$, we get $X_{n} \in\left(\mathfrak{U}_{\infty}\right)_{b}$. Since $\mathfrak{D}_{\infty}(\mathfrak{U}) \subset \mathfrak{D}\left(\left|\overline{T_{\infty}}\right|\right)$, for each $\xi_{\infty} \in \mathfrak{D}_{\infty}(\mathfrak{U})$ we have $\lim _{n \rightarrow \infty} X_{n} \xi_{\infty}=\left|T_{\infty}\right| \xi_{\infty}=\left|T_{\infty}\right| \xi_{\infty}$ and hence $\lim _{n \rightarrow \infty} U_{\infty} X_{n} \xi_{\infty}=U_{\infty}\left|\overline{T_{\infty}}\right| \xi_{\infty}=T_{\infty} \xi_{\infty}$. That is, $U_{\infty} X_{n}$ converges strongly to $T_{\infty}$. Since $\varphi_{\infty}$ and $\psi_{\infty}$ are weakly continuous, we have

$$
\lim _{n \rightarrow \infty} \varphi_{\infty}\left(U_{\infty} X_{n}\right)=\varphi_{\infty}\left(T_{\infty}\right)=\varphi(T)
$$

and $\lim _{n \rightarrow \infty} \psi_{\infty}\left(U_{\infty} X_{n}\right)=\psi_{\infty}(T)=\psi(T)$ and furthermore $\bar{\varphi}=\bar{\psi}$ and $U_{\infty} X_{n} \epsilon$ $\left(\mathscr{U}_{\infty}\right)_{b}$, and so we have $\varphi_{\infty}\left(U_{\infty} X_{n}\right)=\psi_{\infty}\left(U_{\infty} X_{n}\right)$. Therefore we get $\varphi(T)=\psi(T)$.

(2) Suppose $T \in \mathfrak{U}_{h}^{+}$. Then it is easy to show $T_{\infty} \in\left(\mathfrak{U}_{\infty}\right)_{h}^{+}$. Let $\overline{T_{\infty}}=\int_{0}^{\infty} \lambda d E(\lambda)$ be the spectral decomposition of $\bar{T}_{\infty}$ and putting, for each $n, \bar{X}_{n}=\int_{0}^{n} \lambda d E(\lambda)$. By (1), we have $\lim _{n \rightarrow \infty} \varphi_{\infty}\left(X_{n}\right)=\varphi_{\infty}\left(T_{\infty}\right)$. Furthermore, since $\bar{\varphi} \geqq 0$ and $X_{n} \in\left(\mathfrak{N}_{\infty}\right)_{b}^{+}, \varphi_{\infty}\left(X_{n}\right) \geqq 0$ for each $n$. Therefore we get $\varphi(T)=\varphi_{\infty}\left(T_{\infty}\right) \geqq 0$.

Proposition 4.6. Suppose that $\mathfrak{A}$ is a closed $E W^{\sharp}$-algebra on $\mathfrak{D}$ and $f \in \mathfrak{N}_{*}$. Then there exists a couple $(\varphi, U)$ with the following properties;

(a) $\varphi \in \mathfrak{Y}_{*}^{+}$and $\|\bar{\varphi}\|=\|\bar{f}\|$;

(b) $\bar{U}$ is a partial isometry of $\overline{\mathfrak{A}}_{b}$ having $S(\bar{\varphi})$ as the final projection $\bar{U} \bar{U}^{*}=\overline{U U^{\sharp}}$, where $S(\bar{\varphi})$ denotes the support of $\bar{\varphi}$;

(c) $f=\varphi U, \varphi=f U^{\sharp}$;

(d) such decomposition is unique.

Proof. Using Lemma 4.5 and the polar decomposition of a $\sigma$ weakly continuous linear functional $\bar{f}$ on a von Neumann algebra $\overline{\mathfrak{A}}_{b}$, we can easily show Proposition 4.6.

Definition 4.7. The $\varphi$ of Proposition 4.6 is called the absolute value of $f$ and we denote $\varphi$ by $|f|$. This decomposition is called the polar decomposition of $f$.

THEOREM 4.8. Let $\mathfrak{i l}$ be a closed $E W^{\sharp}$-algebra on $\mathfrak{D}$.

( I) The following conditions are equivalent;

(1) $f$ is weakly continuous;

(2) $f=\sum_{i=1}^{n} \omega_{\hat{\xi}_{2}, \eta_{2}}, \xi_{i}, \eta_{i} \in \mathfrak{D}(i=1,2, \cdots, n)$.

(II) The following conditions are equivalent;

(3) $f \in \mathfrak{U}_{*}$; 
(4) $f=\sum_{n=1}^{\infty} \omega_{\xi_{n}, \eta_{n}}, \xi_{\infty}=\left(\xi_{1}, \xi_{2}, \cdots, \xi_{n}, \cdots\right), \eta_{\infty}=\left(\eta_{1}, \cdots\right.$, $\left.\eta_{n}, \cdots\right) \in \mathfrak{D}_{\infty}(\mathfrak{U})$.

Proof. $\quad(2) \Rightarrow(1)$ and $(4) \Rightarrow(3)$; clear.

$(3) \Rightarrow$ (4) Suppose $f \in \mathfrak{A}_{*}$. Let $f=|f| U$ be the polar decomposition of $f$. By Proposition 4.3 there is a $\xi_{\infty}=\left(\xi_{1}, \xi_{2}, \cdots, \xi_{n}, \cdots\right) \in$ $\mathfrak{D}_{\infty}(\mathfrak{U})$ such that $|f|=\sum_{n=1}^{\infty} \omega_{\xi_{n}}$. For each $T \in \mathfrak{U}$ we have

$$
f(T)=(|f| U)(T)=\sum_{n=1}^{\infty}\left(U T \xi_{n} \mid \xi_{n}\right)=\sum_{n=1}^{\infty}\left(T \xi_{n} \mid U^{\sharp} \xi_{n}\right),
$$

and so putting $\eta_{n}=U^{\sharp} \xi_{n}, n=1,2, \cdots, \eta_{\infty}=\left(\eta_{1}, \eta_{2}, \cdots, \eta_{n}, \cdots\right) \in \mathfrak{D}_{\infty}(\mathfrak{U})$ and $f=\sum_{n=1}^{\infty} \omega_{\xi_{n}, \eta_{n}}$.

$(1) \Rightarrow(2)$ By a slight modification of the argument $(3) \Rightarrow(4),(1) \Rightarrow$ (2) is easily shown.

5. The structure of a $\sigma$-weakly continuous homomorphism. In this section we shall show that a $\sigma$-weakly continuous homomorphism of a closed $E W^{\sharp}$-algebra is decomposed in the following three types; a spatial isomorphism, an induction and an amplification.

Definition 5.1. Let $\mathfrak{U}\left(\right.$ resp. $\left.\mathfrak{B}, \mathfrak{B}_{1}\right)$ be a symmetric \#-algebra on $\mathfrak{D}\left(\right.$ resp. $\left.\sqrt{ }, \mathfrak{F}_{1}\right)$. Let $\Phi\left(\right.$ resp. $\left.\Phi_{1}\right)$ be a homomorphism of $\mathfrak{A}$ onto $\mathfrak{B}\left(\right.$ resp. $\left.\mathfrak{B}_{1}\right)$. Then $\Phi$ and $\Phi_{1}$ are called unitarily equivalent if there is an isometric isomorphism $U$ of $\&$ onto $\xi_{1}$ such that

$$
U \Phi(T) \xi=\Phi_{1}(T) U \xi
$$

for all $T \in A$ and $\xi \in \Subset$ and we denote by $\Phi \cong \Phi_{1}$.

LEMMA 5.2. Let $\mathfrak{U}$ be a closed $E W^{\sharp}$-algebra on $\mathfrak{D}$ and $\varphi=\sum_{i=1}^{n} \omega_{\xi_{i}}$, $\xi_{i} \in \mathfrak{D}(i=1, \cdots, n)\left(\operatorname{resp}_{\circ} \varphi=\sum_{i=1}^{\infty} \omega_{\xi_{i}}, \xi_{\infty}=\left(\xi_{1}, \cdots, \xi_{n}, \cdots\right) \in \mathfrak{D}_{\infty}(\mathfrak{U})\right)$. Let $\Re$ be a Hilbert space with dimension $n$ (resp. a separable Hilbert space) and let $\Phi$ be an amplification $T \rightarrow T \widetilde{\otimes} I_{\Re}$ of $\mathfrak{i}$ onto $\mathfrak{\Re} \widetilde{\otimes} I_{\Re}$. Then there exists an element $\rightsquigarrow$ of $\mathfrak{D} \widetilde{\otimes} \Re$ such that $\varphi(T)=(\Phi(T) x \mid x)$ for all $T \in \mathfrak{X}$.

Proof. Suppose that $\left\{e_{2}\right\}_{i=1,2, \ldots}$ is an orthogonal basis in $\Re$. Let $x=\sum_{\imath=1}^{\infty} \xi_{i} \otimes e_{\imath}$. Then we have $\sum_{i=1}^{n} \xi_{i} \otimes e_{i} \rightarrow x(n \rightarrow \infty)$ and

$$
\begin{aligned}
& \left\|\left(T \otimes I_{\Re}\right) \sum_{i=n}^{m} \xi_{i} \otimes e_{i}\right\|^{2}=\left\|\sum_{i=n}^{m} T \xi_{i} \otimes e_{i}\right\|^{2}=\sum_{i=n}^{m}\left\|T \xi_{i}\right\|^{2} \\
& \quad \longrightarrow 0 \quad(n, m \longrightarrow \infty)
\end{aligned}
$$

and hence we get, for all $T \in \mathfrak{A}, x \in \mathscr{D}\left(\overline{T \otimes I_{\Omega}}\right)$ and $\overline{T \otimes I_{\Omega}} x=$ 
$\sum_{i=1}^{\infty} T \xi_{i} \otimes e_{i} . \quad$ That is, $x \in \mathscr{D} \widetilde{\otimes} \Re$ and $\left(T \widetilde{\otimes} I_{\Re}\right) x=\sum_{i=1}^{\infty} T \xi_{i} \otimes e_{i}$. Furthermore we have

$$
\begin{aligned}
(\Phi(T) x \mid x) & =\left(\left(T \widetilde{\otimes} I_{\Omega}\right) x \mid x\right) \\
& =\left(\sum_{i=1}^{\infty} T \xi_{i} \otimes e_{i} \mid \sum_{i=1}^{\infty} \xi_{i} \otimes e_{i}\right)=\sum_{i=1}^{\infty}\left(T \xi_{i} \mid \xi_{i}\right)=\varphi(T) .
\end{aligned}
$$

Let $\mathfrak{A}$ be a closed symmetric \#-algebra on $\mathfrak{D}$ and let $\xi \in \mathfrak{D}$. We denote by $\mathfrak{X}_{\xi}^{\mathfrak{M}}$ the subspace of $\mathfrak{D}$ generated by $\{T \xi ; T \in \mathfrak{X}\}$. Let $\left(\mathfrak{X}_{\xi}^{\mathfrak{M}}\right)^{-}$ be the closure of $\mathfrak{X}_{\xi}^{\mathfrak{q}}$ under the induced topology $\tau_{0}$ and let $E_{\xi}^{\mathfrak{q}}$ be the projection onto $\mathfrak{X}_{\xi}^{\mathfrak{q}}$. Then, by Proposition $3.3, E_{\xi}^{\mathfrak{a}} \in \mathfrak{X}^{\prime}$ and $E_{\xi}^{\mathfrak{a}} \mathfrak{D}=$ $\left(\mathfrak{X}_{\xi}^{\mathfrak{q}}\right)^{-}$.

DEFINITION 5.3. If $\left(\mathfrak{X}_{\xi}^{\mathfrak{q}}\right)^{-}=\mathfrak{D}$, then $\xi$ is called a strongly cyclic vector for $\mathfrak{A}$.

LEMMA 5.4. Let $\mathfrak{U}\left(\operatorname{resp} . \mathfrak{B}, \mathfrak{B}_{1}\right)$ be a closed symmetric \#-algebra on $\mathfrak{D}\left(\right.$ resp. $\left.\mathfrak{F}, \mathfrak{F}_{1}\right)$ and let $\Phi\left(\right.$ resp. $\left.\Phi_{1}\right)$ be a homomorphism of $\mathfrak{A}$ onto $\mathfrak{B}\left(\right.$ resp. $\left.\mathfrak{B}_{1}\right)$. If there is a strongly cyclic vector $\xi \in \mathfrak{E}\left(\right.$ resp. $\left.\xi_{1} \in \xi_{1}\right)$ for $\mathfrak{B}\left(\operatorname{resp} . \mathfrak{B}_{1}\right)$ such that

$$
(\Phi(T) \xi \mid \xi)=\left(\Phi_{1}(T) \xi_{1} \mid \xi_{1}\right)
$$

for all $T \in \mathfrak{N}$, then $\Phi \cong \Phi_{1}$.

Proof. Putting $U_{0} ; \Phi(T) \xi \rightarrow \Phi_{1}(T) \xi_{1}$, we have, for all $T \in \mathfrak{A}$,

$$
\left\|U_{0} \Phi(T) \xi\right\|^{2}=\|\Phi(T) \xi\|^{2},
$$

so that $U_{0}$ is an isometric isomorphism of $\Phi(\mathfrak{H}) \xi$ onto $\Phi_{1}(\mathfrak{Z}) \xi_{1}$ and furthermore, since $\xi\left(\right.$ resp. $\left.\xi_{1}\right)$ is a cyclic vector for $\mathfrak{B}\left(\right.$ resp. $\left.\mathfrak{B}_{1}\right), U_{0}$ is extended to an isometric isomorphism $U$ of $\Re=\overline{\mathcal{E}}$ onto $\Re_{1}=\overline{\mathcal{E}_{1}}$. For each $\eta \in \mathfrak{F}$ there is a net $\left\{T_{\alpha}\right\}$ in $\mathfrak{X}$ such that $\lim _{\alpha} \Phi(T) \Phi\left(T_{\alpha}\right) \xi=\Phi(T) \eta$ for all $T \in \mathfrak{X}$ and then we have $\lim _{\alpha} \Phi_{1}\left(T_{\alpha}\right) \xi_{1}=\lim U \Phi\left(T_{\alpha}\right) \xi=U \eta$ and $\lim _{\alpha} \Phi_{1}(T) \Phi_{1}\left(T_{\alpha}\right) \xi_{1}=\lim _{\alpha} U \Phi(T) \Phi\left(T_{\alpha}\right) \xi=U \Phi(T) \eta$, so that we get $U \eta \epsilon$ $\bigcap_{T \in \mathfrak{r}} \mathfrak{D}\left(\overline{\Phi_{1}(T)}\right)=\mathfrak{F}_{1}$ and $\Phi_{1}(T) U \eta=\overline{\Phi_{1}(T)} U \eta=U \Phi(T) \eta$ for all $T \in \mathfrak{N}$. Similarly we can show $U \mathbb{F} \supset \mathfrak{F}_{1}$ and therefore $\Phi \cong \Phi_{1}$.

THEOREM 5.5. Let $\mathfrak{A}($ resp. $\mathfrak{B})$ be a closed $E W^{\sharp}$-algebra on $\mathfrak{D}($ resp. (5) and let $\Phi$ be a $\sigma$-weakly continuous homomorphism of $\mathfrak{A}$ onto $\mathfrak{B}$. Then there exist an amplification $\Phi_{1}$ of $\mathfrak{i}$ onto a closed $E W^{\sharp}$-algebra $\mathfrak{N}_{1}$ on $\mathfrak{D}_{1}$, an induction $\Phi_{2}$ of $\mathfrak{U}_{1}$ onto a closed $E W^{\sharp}$ algebra $\mathfrak{N}_{2}$ on $\mathfrak{D}_{2}$ and a spatial isomorphism $\Phi_{3}$ of $\mathfrak{X}_{2}$ onto $\mathfrak{B}$ such that $\Phi=\Phi_{3} \circ \Phi_{2} \circ \Phi_{1}$.

Proof. (1) Suppose that $\mathfrak{B}$ has a strongly cyclic vector $\eta \in \mathfrak{F}$. Putting 


$$
\varphi(T)=(\Phi(T) \eta \mid \eta), \quad T \in \mathfrak{X},
$$

$\varphi$ is a $\sigma$-weakly continuous positive linear functional on $\mathfrak{A}$. By Proposition 4.3 there exists a $\xi_{\infty}=\left(\xi_{1}, \xi_{2}, \cdots, \xi_{n}, \cdots\right) \in \mathfrak{D}_{\infty}(\mathfrak{U})$ such that $\varphi=\sum_{i=1}^{\infty} \omega_{\xi_{i}}$. Let $\mathfrak{D}_{1}=\tilde{\mathfrak{D}} \widetilde{\otimes} \Re_{1}\left(\Re_{1}\right.$; a separable Hilbert space), let $\mathfrak{U}_{1}=\mathfrak{U} \widetilde{\otimes} I_{\Re_{1}}$ and let $\Phi_{1}$ be an amlification of $\mathfrak{X}$ onto $\mathfrak{X}_{1}$. By Lemma 5.2 there exists an element $x$ of $\mathfrak{D}_{1}$ such that $\varphi(T)=\left(\Phi_{1}(T) x \mid x\right)$ for all $T \in \mathfrak{A}$. By Proposition $3.11 \mathfrak{2}_{1}$ is a closed $E W^{\sharp}$-algebra on $\mathfrak{D}_{1}$. Let $\mathfrak{D}_{2}=\left(\mathfrak{X}_{x}^{\mathfrak{\alpha}_{1}}\right)^{-}$and let $E=E_{x}^{\mathfrak{x}_{1}}$. Let $\mathfrak{A}_{2}=\left(\mathfrak{U}_{1}\right)_{E}$ and let $\Phi_{2}$ be an induction of $\mathfrak{A}_{1}$ onto $\mathfrak{U}_{2}$. By Theorem $3.5 \mathfrak{A}_{2}$ is a closed $E W^{\sharp}$-algebra on $\mathfrak{D}_{2}$ and

$$
(\Phi(T) \eta \mid \eta)=\varphi(T)=\left(\Phi_{1}(T) x \mid x\right)=\left(\left(\Phi_{2} \circ \Phi_{1}\right)(T) x \mid x\right)
$$

for all $T \in \mathfrak{A}$. Furthermore, $\Phi_{2} \circ \Phi_{1}$ is a homomorphism of $\mathfrak{U}$ onto $\mathfrak{A}_{2}$, $\Phi$ is a homomorphism of $\mathfrak{U}$ onto $\mathfrak{B}$ and $x$ (resp. $\eta$ ) is a strongly cyclic vector for $\mathfrak{U}_{2}(\operatorname{resp}$. $\mathfrak{B})$, so that, by Lemma 5.4, we get $\Phi \cong \Phi_{2} \circ \Phi_{1}$. Putting

$$
\Phi_{3} ; \Phi_{2} \circ \Phi_{1}(T) \longrightarrow \Phi(T), \quad T \in \mathfrak{Z},
$$

$\Phi_{3}$ is a spatial isomorphism of $\mathfrak{U}_{2}$ onto $\mathfrak{B}$. Clearly we have $\Phi=$ $\Phi_{3} \circ \Phi_{2} \circ \Phi_{1}$.

(2) In a general case we shall prove the theorem. Suppose that $\left\{\eta_{c}\right\}_{\in \in A}$ is a maximal family such that $\left\{\eta_{t}\right\}_{c \in \Lambda} \subset \mathbb{F}$ and $\mathbb{F}_{t}=\left(\mathfrak{X}_{\eta_{c}}^{\mathfrak{g}}\right)^{-}$is mutually orthogonal. Let $E_{\iota}=E_{\eta_{c}}^{\mathfrak{9}}$ for every $\iota \in \Lambda$ and then $E_{\iota} \in \mathfrak{B}^{\prime}$ and furthermore we get $\sum_{\iota \in A} E_{c}=I$, by the maximality of $\left\{\eta_{\imath}\right\}_{\iota \in A}$. For each $\iota \in \Lambda$ putting

$$
\mathfrak{B}_{\iota}=\mathfrak{B}_{E_{\iota}} \quad \text { and } \quad \Phi^{\iota}(T)=\Phi(T)_{E \iota}, \quad T \in \mathfrak{A},
$$

$\Phi^{\prime}$ is a $\sigma$-weakly continuous homomorphism and $\mathfrak{B}_{\iota}$ is a closed $E W^{\sharp}$ algebra on $\xi_{\iota}=E_{c} \Subset$ with a strongly cyclic vector $\eta_{\iota}$. By (1), for each $\iota \in \Lambda$, there exist an amplification $\Phi_{1}^{\iota}$ of $\mathfrak{X}$ onto a closed $E W^{\sharp}$ algebra $\mathfrak{N}_{1}^{\iota}=\mathfrak{N} \widetilde{\otimes} I_{\Re_{1}^{\prime}}$ on $\mathfrak{D} \widetilde{\otimes} \Re_{1}^{\iota}$, an induction $\Phi_{2}^{\iota}$ of $\mathfrak{N}_{1}^{\iota}$ onto a closed $E W^{*}$-algebra $\mathfrak{N}_{2}^{c}=\left(\mathfrak{U}_{1}^{\prime}\right)_{F_{t}}\left(F_{c} \in\left(\mathfrak{U}_{1}^{\prime}\right)_{p}^{\prime}\right)$ on $\mathfrak{D}_{2}^{c}=F_{c} \mathfrak{D}_{1}$ and a spatial isomorphism $\Phi_{3}^{\iota}$ of $\mathfrak{U}_{2}^{\prime}$ onto $\mathfrak{B}_{\iota}$ such that $\Phi^{\iota}=\Phi_{3}^{\prime} \circ \Phi_{2}^{\prime} \circ \Phi_{1}^{\prime}$. Let $\Omega_{1}=$ $\bigoplus_{\iota \in \Lambda} \Re_{1}, \mathfrak{N}_{1}=\mathfrak{\imath} \widetilde{\otimes} I_{\Omega_{1}}$ and let $\Phi_{1}$ be an amplification of $\mathfrak{U}$ onto $\mathfrak{N}_{1}$. It

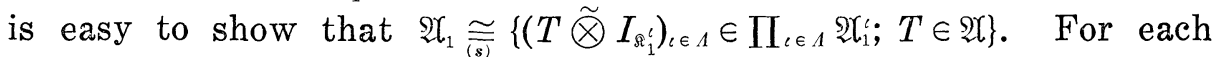
$\iota \in \Lambda$ we have $F_{\iota} \in\left(\mathfrak{Q}_{1}^{\prime}\right)_{p}^{\prime}=\left(\mathfrak{U} \otimes I_{\mathfrak{R}_{1}^{\prime}}^{\prime}\right)_{p}^{\prime}=\left(\mathfrak{U}^{\prime} \otimes \mathscr{B}\left(\Re_{1}^{\imath}\right)\right)_{p}$, so that $F=$ $\left(F_{t}\right)_{i \in A} \in \bigoplus_{i \in A}\left(\mathscr{U}^{\prime} \otimes \mathscr{B}\left(\Re_{1}^{\prime}\right)\right)_{p}=\left(\mathfrak{U}^{\prime} \otimes \mathscr{B}\left(\Re_{1}\right)\right)_{p}=\left(\mathfrak{U}_{1}^{\prime}\right)_{p}$. Let $\mathfrak{A}_{2}=\left(\mathfrak{U}_{1}\right)_{F}$. Then $\mathfrak{U}_{2}$ is a closed $E W^{\sharp}$-algebra on $\mathfrak{D}_{2}=F \mathfrak{D}_{1}$. Let $\Phi_{2}$ be an induction of $\mathfrak{N}_{1}$ onto $\mathfrak{N}_{2}$ and let $\Phi_{3} ; \Phi_{2} \circ \Phi_{1}(T) \rightarrow \Phi(T), T \in \mathfrak{N}$. We shall show that $\Phi_{3}$ is a spatial isomorphism of $\mathfrak{U}_{2}$ onto $\mathfrak{B}$. For each $T \in \mathfrak{N}$ we have 


$$
\begin{aligned}
\Phi_{2} \circ \Phi_{1}(T) & =\left(T \widetilde{\otimes} I_{\Omega_{1}}\right)_{F}=\left(\left(T \widetilde{\otimes} I_{\Omega_{1}^{\prime}}\right)_{\iota \in A}\right)_{(F)_{\iota \in \Lambda}} \\
& =\left(\left(T \widetilde{\otimes} I_{\Omega_{1}}\right)_{F_{c}}\right)_{\iota \in A} \text { (by Proposition 3.10) } \\
& =\left(\left(\Phi_{2}^{\iota} \circ \Phi_{1}^{\iota}\right)(T)\right)_{\iota \in \Lambda} .
\end{aligned}
$$

On the other hand, by Proposition $3.9, \mathfrak{B}$ is spatially isomorphic to $\left\{\left(\Phi^{\prime}(T)\right)_{\iota \in A} \in \prod_{\iota \in A} \mathfrak{B}_{\iota} ; T \in \mathfrak{Q}\right\}$. Furthermore, since $\Phi_{3}^{\iota}$ is a spatial isomorphism for each $\iota \in \Lambda$, we get $\left(\left(\Phi_{2}^{\llcorner} \circ \Phi_{1}^{\iota}\right)(T)\right)_{\iota \in \Lambda} \rightarrow\left(\Phi^{\iota}(T)\right)_{\iota \in \Lambda}$ is a spatial isomorphism, i.e., $\Phi_{3}$ is a spatial isomorphism of $\mathfrak{2}_{2}$ onto $\mathfrak{B}$.

\section{REFERENCES}

1. R. Arens, The space $L^{\omega}$ and convex topological rings, Bull. Amer. Math. Soc., 52 (1946), 931-935.

2. G. R. Allan, On a class of locally convex algebras, Proc. London Math. Soc., (3) 17 (1967), 91-114.

3. J. Diximier, Les algebres d'operateurs dans l'espace hilbertien, Gausthier-Villars, Paris, 2é edition (1969).

4. P. G. Dixon, Generalized $B^{*}$-algebras, Proc. London Math. Soc., (3) 21 (1970), 693715 .

5. U Unbounded operator algebras, Proc. London Math. Soc., (3) 23 (1971), 53-69.

6. — Generalized $B^{*}$-algebas II, J. London Math. Soc., (2) 5 (1972), 159-165.

7. N. Dunford and J. Schwartz, Linear Operators, Vol II, New York; Interscience Pub. (1963).

8. J. M. G. Fell and J. L. Kelley, An algebra of unbounded operators, Proc. Nat. Acad. Sci. U. S. A., (1952), 592-598.

9. A. Inoue, Representations of $G B^{*}$-algebras I, Fukuoka Univ. Sci. Reports, 5 (1975), 63-78.

10. 21-41.

11. R. T. Powers, Self-adjoint algebras of unbounded operators, Commun. Math. Phys., 21 (1971), 85-124.

Received December 17, 1975.

Fukuoka University 



\section{PACIFIC JOURNAL OF MATHEMATICS}

\section{EDITORS}

RICHARD ARENS (Managing Editor) University of California

Los Angeles, California 90024

R. A. Beaumont

University of Washington

Seattle, Washington 98105
J. DUGUNDJI

Department of Mathematics University of Southern Californı

Los Angeles, California 90007

D. Gilbarg and J. Milgram

Stanford University

Stanford, California 94305

\section{ASSOCIATE EDITORS}
E. F. BECKENBACH
B. H. NeumanN
F. WOLF
K. YosHIDA

\section{SUPPORTING INSTITUTIONS}

UNIVERSITY OF BRITISH COLUMBIA

CALIFORNIA INSTITUTE OF TECHNOLOGY

UNIVERSITY OF CALIFORNIA

MONTANA STATE UNIVERSITY

UNIVERSITY OF NEVADA

NEW MEXICO STATE UNIVERSITY

OREGON STATE UNIVERSITY

UNIVERSITY OF OREGON

OSAKA UNIVERSITY
UNIVERSITY OF SOUTHERN CALIFORNIA

STANFORD UNIVERSITY

UNIVERSITY OF TOKYO

UNIVERSITY OF UTAH

WASHINGTON STATE UNIVERSITY

UNIVERSITY OF WASHINGTON

* * * *

AMERICAN MATHEMATICAL SOCIETY

NAVAL WEAPONS CENTER 


\section{Pacific Journal of Mathematics \\ Vol. 65, No. $1 \quad$ September, 1976}

David Lee Armacost, Compactly cogenerated LCA groups ............. 1

Sun Man Chang, On continuous image averaging of probability measures ...... 13

J. Chidambaraswamy, Generalized Dedekind $\psi$-functions with respect to a

polynomial. II................................... 19

Freddy Delbaen, The Dunford-Pettis property for certain uniform algebras ..... 29

Robert Benjamin Feinberg, Faithful distributive modules over incidence

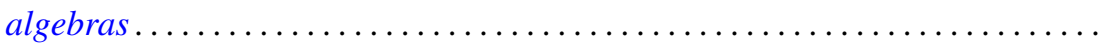

Paul Froeschl, Chained rings . . . . . . . . . . . . . . . . . . . .

John Brady Garnett and Anthony G. O'Farrell, Sobolev approximation by a sum

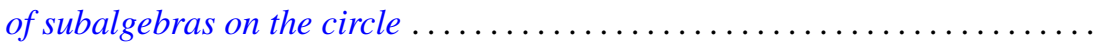

Hugh M. Hilden, José M. Montesinos and Thomas Lusk Thickstun, Closed

oriented 3-manifolds as 3-fold branched coverings of $S^{3}$ of special type.....

Atsushi Inoue, On a class of unbounded operator algebras ................

Peter Kleinschmidt, On facets with non-arbitrary shapes.

Narendrakumar Ramanlal Ladhawala, Absolute summability of Walsh-Fourier

series

Howard Wilson Lambert, Links which are unknottable by maps . . . . . . . . . . .

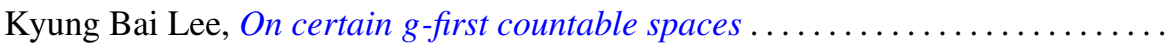

Richard Ira Loebl, A Hahn decomposition for linear maps .................

Moshe Marcus and Victor Julius Mizel, A characterization of functionals on $W_{1}^{p}$ possessing autonomous kernels. I . .

James Miller, Subordinating factor sequences and convex functions of several

variables.

Keith Pierce, Amalgamated sums of abelian l-groups ...

Jonathan Rosenberg, The $C^{*}$-algebras of some real and $p$-adic solvable

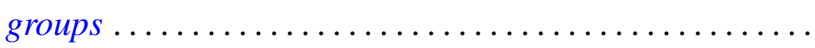

Hugo Rossi and Michele Vergne, Group representations on Hilbert spaces defined

in terms of $\partial_{b}$-cohomology on the Silov boundary of a Siegel domain . .

Mary Elizabeth Schaps, Nonsingular deformations of a determinantal

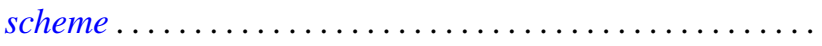

S. R. Singh, Some convergence properties of the Bubnov-Galerkin method...

Peggy Strait, Level crossing probabilities for a multi-parameter Brownian

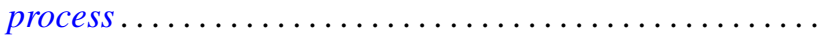

Robert M. Tardiff, Topologies for probabilistic metric spaces .

Benjamin Baxter Wells, Jr., Rearrangements of functions on the ring of integers of

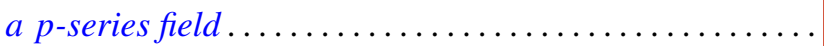

Robert Francis Wheeler, Well-behaved and totally bounded approximate identities for $C_{0}(X)$.

Delores Arletta Williams, Gauss sums and integral quadratic forms over local

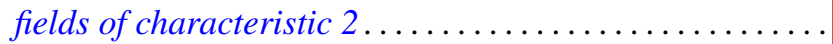

John Yuan, On the construction of one-parameter semigroups in topological 